\title{
Divergence-Free Wavelets on the Hypercube: General Boundary Conditions
}

\author{
Rob Stevenson ${ }^{1}$
}

Received: 8 January 2015 / Revised: 28 November 2015 / Accepted: 11 December 2015

Published online: 16 February 2016

(C) The Author(s) 2016. This article is published with open access at Springerlink.com

\begin{abstract}
On the $n$-dimensional hypercube, for given $k \in \mathbb{N}$, wavelet Riesz bases are constructed for the subspace of divergence-free vector fields of the Sobolev space $H^{k}\left((0,1)^{n}\right)^{n}$ with general homogeneous Dirichlet boundary conditions, including slip or no-slip boundary conditions. Both primal and suitable dual wavelets can be constructed to be locally supported. The construction of the isotropic wavelet bases is restricted to the square, but that of the anisotropic wavelet bases applies for any space dimension $n$.
\end{abstract}

Keywords Divergence-free wavelets · Biorthogonal space decompositions · Riesz bases - Anisotropic wavelets · Compactly supported wavelets

Mathematics Subject Classification 42C40 - 65T60 - 76D99

\section{Introduction}

\subsection{Overview}

This paper concerns the construction of a Riesz basis, consisting of wavelets, for the space

Communicated by Wolfgang Dahmen.

$\triangle$ Rob Stevenson

R.P.Stevenson@uva.nl

1 Korteweg-de Vries Institute for Mathematics, University of Amsterdam, P.O. Box 94248, 1090 GE Amsterdam, The Netherlands 


$$
\mathbf{H}_{0}(\operatorname{div} 0 ; \Omega):=\left\{\mathbf{v} \in L_{2}(\Omega)^{n}: \operatorname{div} \mathbf{v}=0, \mathbf{v} \cdot \mathbf{n}=0 \text { on } \partial \Omega\right\}
$$

where $\Omega \subset \mathbb{R}^{n}$, or for this space intersected with $H^{k}(\Omega)^{n}$, or with some closed subspace of the latter that incorporates additional boundary conditions. We take $\Omega$ to be the hypercube $\mathcal{I}^{n}$, where $\mathcal{I}:=(0,1)$. The construction can be transferred to other bounded domains by means of the Piola transform.

Divergence-free wavelet bases find their applications in approximating the solution of the incompressible (Navier-) Stokes equations. They can be used either for solving these equations, see, e.g., [9,26,29], or for analyzing, or finding efficient representations, of approximate solutions that were obtained by other means, see, e.g., $[7,28]$.

Battle and Federbush [2] were the first who constructed an orthogonal wavelet basis for $\mathbf{H}_{0}\left(\operatorname{div} 0 ; \mathbb{R}^{n}\right)=\mathbf{H}\left(\operatorname{div} 0 ; \mathbb{R}^{n}\right):=\left\{\mathbf{v} \in L_{2}\left(\mathbb{R}^{n}\right)^{n}: \operatorname{div} \mathbf{v}=0\right\}$. These wavelets were globally supported and therefore cannot be applied on bounded domains.

The construction by Lemarié-Rieusset in [21] of a wavelet Riesz basis for $\mathbf{H}\left(\operatorname{div} 0 ; \mathbb{R}^{n}\right)$ relies on the availability of two pairs of biorthogonal Riesz bases $(\Psi, \tilde{\Psi})$ and $(\Psi, \widetilde{\Psi})$, both for the pair $\left(L_{2}(\mathbb{R}), L_{2}(\mathbb{R})\right)$, that, for some invertible diagonal matrix D, satisfy

$$
\Psi^{\prime}=\mathbf{D} \Psi, \quad \tilde{\Psi}^{\prime}=-\mathbf{D} \tilde{\Psi}
$$

(bases are formally viewed as (infinite) column vectors). Compactly supported primal and dual wavelets that satisfy these conditions were constructed. Having such collections of univariate wavelets at hand, the coordinate functions of the divergence-free wavelets are constructed as tensor products of the univariate wavelets (and possibly scaling functions).

The construction from [21] on $\mathbb{R}^{n}$ can be mimicked on $\mathcal{I}^{n}$ once one has pairs of biorthogonal Riesz bases $(\Psi, \tilde{\Psi})$ and $(\Psi, \bar{\Psi})$, now for $\left(L_{2}(\mathcal{I}), L_{2}(\mathcal{I})\right)$, that satisfy (1.1). Quite a few papers have been devoted to this approach, see, e.g., [8, 14, 15, 19,27]. It seems, however, that results fully analogous to those on $\mathbb{R}^{n}$ have not been realized for $n \geq 3$. The reason for this can be understood as follows: Integration by parts shows

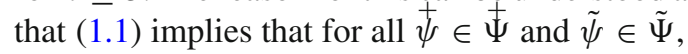

$$
\dot{+}(1) \tilde{\psi}(1)-+\dot{\psi}(0) \tilde{\psi}(0)=0
$$

To obtain such vanishing boundary terms, one may consider, e.g., $\stackrel{+}{\Psi} \subset H_{0}^{1}(\mathcal{I})$. Yet, then any element of $\Psi=\mathbf{D}^{-1} \Psi^{\prime}$ has a vanishing mean, so that $\Psi$ can only be a basis for $L_{2,0}(\mathcal{I}):=\left\{u \in L_{2}(\mathcal{I}): \int_{\mathcal{I}} u=0\right\}$. When one ignores this defect and follows the construction from [21], one ends up with a Riesz basis for

$$
\mathbf{H}_{0}\left(\operatorname{div} 0 ; \mathcal{I}^{n}\right) \cap \mathbf{L}_{2,0}\left(\mathcal{I}^{n}\right),
$$

where

$$
\begin{aligned}
\mathbf{L}_{2,0}\left(\mathcal{I}^{n}\right):= & L_{2}(\mathcal{I}) \otimes L_{2,0}(\mathcal{I}) \otimes \cdots \otimes L_{2,0}(\mathcal{I}) \\
& \times \cdots \times L_{2,0}(\mathcal{I}) \otimes \cdots \otimes L_{2,0}(\mathcal{I}) \otimes L_{2}(\mathcal{I})
\end{aligned}
$$


'Coincidentally', it holds that $\mathbf{H}_{0}\left(\operatorname{div} 0 ; \mathcal{I}^{2}\right) \cap \mathbf{L}_{2,0}\left(\mathcal{I}^{2}\right)=\mathbf{H}_{0}\left(\operatorname{div} 0 ; \mathcal{I}^{2}\right)$, so that a Riesz basis for $\mathbf{H}_{0}\left(\operatorname{div} 0 ; \mathcal{I}^{2}\right)$ is obtained. For $n \geq 3$, however, $\mathbf{H}_{0}\left(\operatorname{div} 0 ; \mathcal{I}^{n}\right) \cap \mathbf{L}_{2,0}\left(\mathcal{I}^{n}\right)$ is a true subspace of $\mathbf{H}_{0}\left(\operatorname{div} 0 ; \mathcal{I}^{n}\right)$, with a co-dimension that is infinite.

To solve this problem, in [25] we made an orthogonal decomposition of $L_{2}\left(\mathcal{I}^{n}\right)^{n}$ into $2^{n}-1$ subspaces, each of them being isomorphic to $\mathbf{L}_{2,0}\left(\mathcal{I}^{\ell}\right)$ for some $\ell=$ $1, \ldots, n$. Using the approach from [21], the divergence-free parts of each of these subspaces could be equipped with wavelet bases. Moreover, the union of these bases was shown to be a Riesz basis for $\mathbf{H}_{0}\left(\operatorname{div} 0 ; \mathcal{I}^{n}\right)$.

Since functions in the aforementioned subspaces have components that are constants as function of some variables, the divergence-free wavelets that were obtained do not satisfy boundary conditions beyond having vanishing normals. Consequently, this construction was restricted to slip boundary conditions.

The aim of the current paper is to extend the approach to general boundary conditions, including no-slip boundary conditions. The key to achieve this will be the replacement of the orthogonal decomposition of $L_{2}\left(\mathcal{I}^{n}\right)^{n}$ into $2^{n}-1$ subspaces, by a biorthogonal decomposition of $\left(L_{2}\left(\mathcal{I}^{n}\right)^{n}, L_{2}\left(\mathcal{I}^{n}\right)^{n}\right)$ into $2^{n}-1$ pairs of subspaces. With this approach, we will be able to construct a wavelet Riesz basis for $\mathbf{H}_{0}\left(\operatorname{div} 0 ; \mathcal{I}^{n}\right)$ that for given $k$, renormalized, will be a basis for $\mathbf{H}^{k}\left(\mathcal{I}^{n}\right) \cap \mathbf{H}_{0}\left(\operatorname{div} 0 ; \mathcal{I}^{n}\right)$, with the first space being the closed subspace of $H^{k}\left(\mathcal{I}^{n}\right)^{n}$ defined by imposing (very) general homogeneous Dirichlet boundary conditions up to order $k$.

\subsection{The Construction from [21]}

To further explain the difficulties and possibilities with transferring the construction of a divergence-free wavelet basis on $\mathbb{R}^{n}$ from [21] to one on a hypercube, first we describe it in some detail. To appreciate the steps that will be taken in the following sections in an abstract framework, we expect that the reader will find it useful to go through this description and the remainder of the introduction.

For ease of presentation, in this subsection we consider $n=2$, and, although in [21] isotropic bivariate wavelets are constructed, we consider the construction of anisotropic bivariate wavelets. With an isotropic construction, a bivariate wavelet is the tensor product of two univariate functions on the same scale, either both wavelets or a wavelet and a scaling function. With an anisotropic construction, a bivariate wavelet is the tensor product of two univariate wavelets on arbitrary, unrelated scales. The idea to construct anisotropic divergence-free wavelets originates from [8].

We write $\Psi=\left\{\psi_{\lambda}: \lambda \in \nabla\right\}$, with $\nabla$ being a countable index set, and similarly for the other three collections from (1.1), and $\mathbf{D}=\operatorname{diag}\left[d_{\lambda}\right]_{\lambda \in \nabla}$. From $L_{2}\left(\mathbb{R}^{2}\right) \simeq$ $L_{2}(\mathbb{R}) \otimes L_{2}(\mathbb{R})$, and the fact that all four collections are Riesz bases for $L_{2}(\mathbb{R})$, we infer that

$$
\begin{array}{r}
\bigcup_{\lambda, \mu \in \nabla}\left\{\dot{\psi}_{\lambda} \otimes \psi_{\mu} \mathbf{e}_{1}, \psi_{\lambda} \otimes \dot{\psi}_{\mu} \mathbf{e}_{2}\right\}, \\
\bigcup_{\lambda, \mu \in \nabla}\left\{\tilde{\psi}_{\lambda} \otimes \tilde{\psi}_{\mu} \mathbf{e}_{1}, \tilde{\psi}_{\lambda} \otimes \tilde{\psi}_{\mu} \mathbf{e}_{2}\right\}
\end{array}
$$

are biorthogonal Riesz bases for $\left(L_{2}\left(\mathbb{R}^{2}\right)^{2}, L_{2}\left(\mathbb{R}^{2}\right)^{2}\right)$. That is, denoting the elements of the first (primal) and second (dual) basis as $\sigma_{\lambda, \mu, i}$ and $\tilde{\sigma}_{\lambda, \mu, i}$, where $i \in\{1,2\}$, 
it holds that $\left\langle\sigma_{\lambda, \mu, i}, \tilde{\sigma}_{\lambda^{\prime}, \mu^{\prime}, i^{\prime}}\right\rangle_{L_{2}\left(\mathbb{R}^{2}\right)^{2}}=1$ when $(\lambda, \mu, i)=\left(\lambda^{\prime}, \mu^{\prime}, i^{\prime}\right)$, and it is zero otherwise.

$$
\begin{aligned}
& \text { Using that } \frac{1}{\sqrt{d_{\lambda}^{2}+d_{\mu}^{2}}}\left[\begin{array}{cc}
-d_{\mu} & d_{\lambda} \\
d_{\lambda} & d_{\mu}
\end{array}\right] \text { is an orthogonal matrix, we find that also } \\
& \bigcup_{\lambda, \mu \in \nabla}\left\{\frac{-d_{\mu} \dot{\psi}_{\lambda} \otimes \psi_{\mu} \mathbf{e}_{1}+d_{\lambda} \psi_{\lambda} \otimes \stackrel{+}{\psi}_{\mu} \mathbf{e}_{2}}{\sqrt{d_{\lambda}^{2}+d_{\mu}^{2}}}, \frac{d_{\lambda} \stackrel{+}{\psi}_{\lambda} \otimes \psi_{\mu} \mathbf{e}_{1}+d_{\mu} \psi_{\lambda} \otimes \stackrel{\psi}{\psi}_{\mu} \mathbf{e}_{2}}{\sqrt{d_{\lambda}^{2}+d_{\mu}^{2}}}\right\}, \\
& \bigcup_{\lambda, \mu \in \nabla}\left\{\frac{-d_{\mu} \tilde{\psi}_{\lambda} \otimes \tilde{\psi}_{\mu} \mathbf{e}_{1}+d_{\lambda} \tilde{\psi}_{\lambda} \otimes \tilde{\psi}_{\mu} \mathbf{e}_{2}}{\sqrt{d_{\lambda}^{2}+d_{\mu}^{2}}}, \frac{d_{\lambda} \tilde{\psi}_{\lambda} \otimes \tilde{\psi}_{\mu} \mathbf{e}_{1}+d_{\mu} \tilde{\psi}_{\lambda} \otimes \tilde{\psi}_{\mu} \mathbf{e}_{2}}{\sqrt{d_{\lambda}^{2}+d_{\mu}^{2}}}\right\}
\end{aligned}
$$

are biorthogonal Riesz bases for $\left(L_{2}\left(\mathbb{R}^{2}\right)^{2}, L_{2}\left(\mathbb{R}^{2}\right)^{2}\right)$. By the crucial relation (1.1), the first element of each couple of primal basis functions is divergence-free, and the second element of each couple of dual basis functions is equal to $\frac{-1}{\sqrt{d_{\lambda}^{2}+d_{\mu}^{2}}} \operatorname{grad} \tilde{\psi}_{\lambda} \otimes \tilde{\psi}_{\mu} \in$ $\operatorname{grad} H^{1}\left(\mathbb{R}^{2}\right)$. So when writing $\mathbf{v} \in \mathbf{H}\left(\operatorname{div} 0 ; \mathbb{R}^{2}\right) \subset L_{2}\left(\mathbb{R}^{2}\right)^{2}$ w.r.t. the primal basis, the coefficient in front of the second element of any couple, being an inner product of $\mathbf{v}$ with the second element of the corresponding dual couple, vanishes because of $\mathbf{H}\left(\operatorname{div} 0 ; \mathbb{R}^{2}\right) \perp_{L_{2}\left(\mathbb{R}^{2}\right)^{2}} \operatorname{grad} H^{1}\left(\mathbb{R}^{2}\right)$. Together, both observations imply that

$$
\bigcup_{\lambda, \mu \in \nabla}\left\{\frac{-d_{\mu} \dot{\psi}_{\lambda} \otimes \psi_{\mu} \mathbf{e}_{1}+d_{\lambda} \psi_{\lambda} \otimes \stackrel{+}{\psi}_{\mu} \mathbf{e}_{2}}{\sqrt{d_{\lambda}^{2}+d_{\mu}^{2}}}\right\} \text { is a Riesz basis for } \mathbf{H}\left(\operatorname{div} 0 ; \mathbb{R}^{2}\right),
$$

with a dual basis given by $\bigcup_{\lambda, \mu \in \nabla}\left\{\frac{d_{\lambda} \tilde{\psi}_{\lambda} \otimes \tilde{\psi}_{\mu} \mathbf{e}_{1}+d_{\mu} \tilde{\psi}_{\lambda} \otimes \tilde{\psi}_{\mu} \mathbf{e}_{2}}{\sqrt{d_{\lambda}^{2}+d_{\mu}^{2}}}\right\}$.

Moreover, taking $\Psi$ and (thus) $\stackrel{+}{\Psi}$ such that, renormalized, they are Riesz bases for $H^{k}(\mathbb{R})$ and $H^{k+1}(\mathbb{R})$, respectively, then, renormalized, the collection from (1.6) is a Riesz basis for $H^{k}\left(\mathbb{R}^{2}\right)^{2} \cap \mathbf{H}\left(\operatorname{div} 0 ; \mathbb{R}^{2}\right)$. In particular, the case $k=1$ is most relevant for the application in solving flow problems. This construction of a divergence-free wavelet basis generalizes to $\mathbb{R}^{n}$ for $n \geq 2$.

Note that here, and similarly in the remainder of the paper, for $\mathbf{X} \subset L_{2}\left(\mathbb{R}^{n}\right)^{n}$ and $\boldsymbol{\Sigma}$ being a Riesz basis for $\mathbf{X} \cap \mathbf{H}\left(\operatorname{div} 0 ; \mathbb{R}^{n}\right)$, we call $\tilde{\boldsymbol{\Sigma}} \subset \mathbf{X}^{\prime}$ a dual basis when

$$
\tilde{\mathbf{\Sigma}}(\boldsymbol{\Sigma})=\mathrm{Id} \text {, and } \mathbf{v} \mapsto \tilde{\boldsymbol{\Sigma}}(\mathbf{v}) \in B\left(\mathbf{X}, \ell_{2}(\tilde{\mathbf{\Sigma}})\right) \text {. }
$$

So, in view of some nonexistence results proven in [20,22], we do not impose that $\tilde{\boldsymbol{\Sigma}} \subset \mathbf{H}\left(\operatorname{div} 0 ; \mathbb{R}^{n}\right)$. Note that nevertheless, $\mathbf{X} \rightarrow \mathbf{X}: \mathbf{v} \mapsto \tilde{\boldsymbol{\Sigma}}(\mathbf{v})^{\top} \boldsymbol{\Sigma}$ is an oblique, and by the second property in (1.7), bounded projector onto $\mathbf{X} \cap \mathbf{H}\left(\operatorname{div} 0 ; \mathbb{R}^{n}\right)$.

For completeness, with $\tilde{\boldsymbol{\Sigma}}(\mathbf{v})$ and $\tilde{\boldsymbol{\Sigma}}(\boldsymbol{\Sigma})$, we mean the infinite column vector $[\tilde{\boldsymbol{\sigma}}(\mathbf{v})]_{\tilde{\boldsymbol{\sigma}} \in \tilde{\boldsymbol{\Sigma}}}$, and the bi-infinite matrix $[\tilde{\boldsymbol{\sigma}}(\boldsymbol{\sigma})]_{\tilde{\boldsymbol{\sigma}} \in \tilde{\boldsymbol{\Sigma}}, \boldsymbol{\sigma} \in \boldsymbol{\Sigma}}\left(\right.$ also denoted by $\langle\tilde{\boldsymbol{\Sigma}}, \boldsymbol{\Sigma}\rangle_{L_{2}\left(\mathbb{R}^{n}\right)^{n}}$ when $\left.\tilde{\Sigma} \subset L_{2}\left(\mathbb{R}^{n}\right)^{n}\right)$.

For the application of a wavelet basis $\Sigma$ for solving an operator equation, in this setting typically a flow problem, the availability of a corresponding dual basis $\tilde{\Sigma}$ is 
not required. For other applications of divergence-free wavelets, as the analysis or compression of earlier computed approximate solutions, explicit knowledge of a dual basis is essential. Moreover, for efficient implementations of such applications, it is needed that, as the primal functions, the dual functions are locally supported.

\subsection{Difficulties with Transferring the Construction from $\mathbb{R}^{n}$ to $\mathcal{I}^{n}$}

The key is to have available two pairs of biorthogonal Riesz bases $(\Psi, \tilde{\Psi})$ and $(\dot{\Psi}, \widetilde{\Psi})$, now for $\left(L_{2}(\mathcal{I}), L_{2}(\mathcal{I})\right)$, that for some invertible diagonal matrix $\mathbf{D}$, satisfy (1.1). Under these assumptions, integration by parts shows that the bi-infinite matrix

$$
\begin{aligned}
& \left.\left.\dot{\Psi}\right|_{x=1} \tilde{\Psi}\right|_{x=1} ^{\top}-\left.\left.\dot{\Psi}\right|_{x=0} \tilde{\Psi}\right|_{x=0} ^{\top}=\left\langle\dot{\Psi}^{\prime}, \tilde{\Psi}\right\rangle_{L_{2}(\mathcal{I})}+\left\langle\dot{\Psi}, \tilde{\Psi}^{\prime}\right\rangle_{L_{2}(\mathcal{I})} \\
& \quad=\langle\mathbf{D} \Psi, \tilde{\Psi}\rangle_{L_{2}(\mathcal{I})}-\langle\dot{\Psi}, \mathbf{D} \tilde{\Psi}\rangle_{L_{2}(\mathcal{I})}=\mathbf{D} \circ \mathrm{Id}-\mathrm{Id} \circ \mathbf{D}=0
\end{aligned}
$$

i.e., as has been claimed before, necessarily,

$$
\stackrel{+}{\psi}(1) \tilde{\psi}(1)-\stackrel{+}{\psi}(0) \tilde{\psi}(0)=0 \quad(\stackrel{+}{\psi} \in \stackrel{+}{\Psi}, \tilde{\psi} \in \tilde{\Psi}) .
$$

To obtain such vanishing boundary terms, one may consider $\stackrel{+}{\Psi} \subset H_{0}^{1}(\mathcal{I})$. Yet, then any element of $\Psi=\mathbf{D}^{-1} \Psi^{\prime}$ has a vanishing mean, so that $\Psi$ cannot be a basis for $L_{2}(\mathcal{I})$, the reason being that the mean value is a nonzero, continuous functional on $L_{2}(\mathcal{I})$. (Taking the mean value is not continuous on $L_{2}(\mathbb{R})$, and therefore the latter space can be equipped with a Riesz basis of functions all having a vanishing mean. This difference can be seen as the main reason for the difficulties of transporting the construction of a divergence-free wavelet basis on $\mathbb{R}^{n}$ to the hypercube.)

Alternatively, we may search $\tilde{\Psi}$ in $H_{0}^{1}(\mathcal{I})$. In this case, the same argument shows that $\tilde{\Psi}$ cannot be a basis for $L_{2}(\mathcal{I})$, and so neither can $\dot{\Psi}$, and we end up with the same problem.

A third possibility is to impose periodic boundary conditions for both $\stackrel{+}{\Psi}$ and $\tilde{\Psi}$. In this case, any element from even both $\Psi$ and $\widetilde{\Psi}$ has vanishing mean, giving rise to the same problem as above.

Finally, a valid choice, which was made in [24], is to search Riesz bases $\tilde{\Psi}$ and $\dot{\Psi}$ for $L_{2}(\mathcal{I})$ such that the elements of $\tilde{\Psi}$ vanish at 1 and those of $\Psi$ vanish at 0 . With this choice, (1.1) can be satisfied, and with that a divergence-free wavelet Riesz basis can be constructed on $\mathcal{I}^{n}$. In view of (1.3), it is, however, a basis for $\left\{\mathbf{v} \in L_{2}(\mathcal{I})^{n}: \operatorname{div} \mathbf{v}=\right.$ $0, \mathbf{v} \cdot \mathbf{n}=0$ on $\left.\partial \mathbb{R}_{+}^{n}\right\}$, being the space of divergence-free functions on $\mathcal{I}^{n}$ subject to the unusual boundary condition of having vanishing normal components on half of the boundary of $\mathcal{I}^{n}$.

A seemingly related solution was found in [18], where a wavelet basis was constructed for $\mathbf{H}_{0}\left(\operatorname{div} 0 ; \mathbb{R}_{+}^{2}\right)$.

\subsection{A Remedy}

Taking $\stackrel{+}{\Psi} \subset H_{0}^{1}(\mathcal{I})$, the condition (1.1) can be satisfied for $\stackrel{+}{\Psi}, \tilde{\Psi}, \bar{\Psi}$ being Riesz bases for $L_{2}(\mathcal{I})$, and $\Psi$ being a Riesz basis for $L_{2,0}(\mathcal{I})$. Then, similarly to (1.3), 
$\bigcup_{\lambda}\left\{\stackrel{\psi}{\psi}_{\lambda_{1}} \otimes \psi_{\lambda_{2}} \otimes \cdots \otimes \psi_{\lambda_{n}} \mathbf{e}_{1}, \ldots, \psi_{\lambda_{1}} \otimes \cdots \otimes \psi_{\lambda_{n-1}} \otimes \stackrel{+}{\psi}_{\lambda_{n}} \mathbf{e}_{n}\right\}$

is a Riesz basis for $\mathbf{L}_{2,0}\left(\mathcal{I}^{n}\right)$. Now when from (1.9) a collection of divergence-free wavelets is constructed, similarly as (1.6) was constructed from (1.3), then this collection will be a Riesz basis for $\mathbf{H}_{0}\left(\operatorname{div} 0 ; \mathcal{I}^{n}\right) \cap \mathbf{L}_{2,0}\left(\mathcal{I}^{n}\right)$.

In [25], this fact was employed as follows: Consider the orthogonal decomposition

$$
L_{2}=L_{2,0} \oplus^{\perp} \llbracket \mathbb{1} \rrbracket,
$$

where $\mathbb{1}:=x \mapsto 1$, and where we used the shorthand notation $L_{2}:=L_{2}(\mathcal{I}), L_{2,0}:=$ $L_{2,0}(\mathcal{I})$, and $\llbracket \cdot \rrbracket:=\operatorname{span}\{\cdot\}$. It gives rise to an orthogonal decomposition of $L_{2}\left(\mathcal{I}^{n}\right)^{n}$ into $2^{n}-1$ subspaces, $\left(\begin{array}{l}n \\ \ell\end{array}\right)$ of them, for $\ell=1, \ldots, n$, being isomorphic to $\mathbf{L}_{2,0}\left(\mathcal{I}^{\ell}\right)$. For example, for $n=3$, this decomposition into 7 subspaces reads as follows:

$$
\begin{aligned}
& L_{2}\left(\mathcal{I}^{3}\right)^{3}=L_{2} \otimes L_{2,0} \otimes L_{2,0} \times L_{2,0} \otimes L_{2} \otimes L_{2,0} \times L_{2,0} \otimes L_{2,0} \otimes L_{2}\left(=\mathbf{L}_{2,0}\left(\mathcal{I}^{3}\right)\right) \\
& \oplus^{\perp} L_{2} \otimes L_{2,0} \otimes \llbracket \mathbb{1} \rrbracket \times L_{2,0} \otimes L_{2} \otimes \llbracket \mathbb{1} \rrbracket \times \quad 0 \quad\left(\simeq \mathbf{L}_{2,0}\left(\mathcal{I}^{2}\right)\right) \\
& \oplus^{\perp} L_{2} \otimes \llbracket \mathbb{1} \rrbracket \otimes L_{2,0} \times \quad 0 \quad \times L_{2,0} \otimes \llbracket \mathbb{1} \rrbracket \otimes L_{2}\left(\simeq \mathbf{L}_{2,0}\left(\mathcal{I}^{2}\right)\right) \\
& \oplus^{\perp} \quad 0 \quad \times \llbracket \mathbb{1} \rrbracket \otimes L_{2} \otimes L_{2,0} \times \llbracket \mathbb{1} \rrbracket \otimes L_{2,0} \otimes L_{2}\left(\simeq \mathbf{L}_{2,0}\left(\mathcal{I}^{2}\right)\right) \\
& \oplus^{\perp} L_{2} \otimes \llbracket \mathbb{1} \rrbracket \otimes \llbracket \mathbb{1} \rrbracket \times \quad 0 \quad \times \quad 0 \quad 0 \quad\left(\simeq \mathbf{L}_{2,0}(\mathcal{I})\right) \\
& \oplus^{\perp} \quad 0 \quad \times \llbracket \mathbb{1} \rrbracket \otimes L_{2} \otimes \llbracket \mathbb{1} \rrbracket \times \quad 0 \quad\left(\simeq \mathbf{L}_{2,0}(\mathcal{I})\right) \\
& \oplus^{\perp} \quad 0 \quad \times \quad 0 \quad \times \llbracket \mathbb{1} \rrbracket \otimes \llbracket \mathbb{1} \rrbracket \otimes L_{2}\left(\simeq \mathbf{L}_{2,0}(\mathcal{I})\right)
\end{aligned}
$$

(note $L_{2}(\mathcal{I})=\mathbf{L}_{2,0}(\mathcal{I})$ ). The isomorphisms between the spaces $\mathbf{L}_{2,0}\left(\mathcal{I}^{\ell}\right)$ and the corresponding subspaces in this decomposition are of the simple type of adding $n-\ell$ zero coordinates to an $\ell$-dimensional vector field, and extending the nonzero coordinate functions as a constant function of the additional $n-\ell$ variables.

Building on the approach from [21], we can equip each of the spaces $\mathbf{L}_{2,0}\left(\mathcal{I}^{\ell}\right)$, or more precisely, each pair $\left(\mathbf{L}_{2,0}\left(\mathcal{I}^{\ell}\right), \mathbf{L}_{2,0}\left(\mathcal{I}^{\ell}\right)\right)$, with biorthogonal Riesz bases that split into two parts, the primals from the first part being divergence-free with vanishing normals at the boundary, and the duals from the second part being gradient fields. The embeddings of the spaces $\mathbf{L}_{2,0}\left(\mathcal{I}^{\ell}\right)$ into $L_{2}\left(\mathcal{I}^{n}\right)^{n}$ preserve the properties of a vector field being divergence-free and having a vanishing normal at the boundary, or, at the dual side, being a gradient field. Consequently, we obtain biorthogonal Riesz bases for $\left(L_{2}\left(\mathcal{I}^{n}\right)^{n}, L_{2}\left(\mathcal{I}^{n}\right)^{n}\right)$ that split into two parts, the primals from the first part being divergence-free with vanishing normals at the boundary, and the duals from the second part being gradient fields. Together both observations imply that the primals from the first part form a Riesz basis for $\mathbf{H}_{0}\left(\operatorname{div} 0 ; \mathcal{I}^{n}\right)$.

By applying suitable univariate biorthogonal wavelet bases, which serve as a building block of this construction, simultaneously one obtains a basis for $H^{k}\left(\mathcal{I}^{n}\right)^{n} \cap$ $\mathbf{H}_{0}\left(\operatorname{div} 0 ; \mathcal{I}^{n}\right)$ for a range of $k$, being most relevant for $k=1$.

Remark 1.1 The aforementioned splitting of the biorthogonal bases for $\left(\mathbf{L}_{2,0}\left(\mathcal{I}^{\ell}\right), \mathbf{L}_{2,0}\left(\mathcal{I}^{\ell}\right)\right)$ into two parts yields for $\ell=1$ a first part that is empty (indeed, 
$u^{\prime}=0$ on $\mathcal{I}$, and $u=0$ on $\partial \mathcal{I}$ implies $u=0$ ). As a consequence, in view of

$$
L_{2}\left(\mathcal{I}^{2}\right)^{2}=\underbrace{L_{2} \otimes L_{2,0} \times L_{2,0} \otimes L_{2}}_{\mathbf{L}_{2,0}\left(\mathcal{I}^{2}\right)} \oplus^{\perp} \underbrace{L_{2} \otimes \llbracket 1 \rrbracket \times 0}_{\simeq \mathbf{L}_{2,0}(\mathcal{I})} \oplus^{\perp} \underbrace{0 \times \llbracket 1 \rrbracket \otimes L_{2}}_{\simeq \mathbf{L}_{2,0}(\mathcal{I})},
$$

we have $\mathbf{H}_{0}\left(\operatorname{div} 0 ; \mathcal{I}^{2}\right)=\mathbf{H}_{0}\left(\operatorname{div} 0 ; \mathcal{I}^{2}\right) \cap \mathbf{L}_{2,0}\left(\mathcal{I}^{2}\right)$. For the cube, however, (1.10) shows that $\mathbf{H}_{0}\left(\operatorname{div} 0 ; \mathcal{I}^{3}\right) \simeq \mathbf{H}_{0}\left(\operatorname{div} 0 ; \mathcal{I}^{3}\right) \cap \mathbf{L}_{2,0}\left(\mathcal{I}^{3}\right) \times \mathbf{H}_{0}\left(\operatorname{div} 0 ; \mathcal{I}^{2}\right)^{3}$, which confirms that the co-dimension of $\mathbf{H}_{0}\left(\operatorname{div} 0 ; \mathcal{I}^{3}\right) \cap \mathbf{L}_{2,0}\left(\mathcal{I}^{3}\right)$ in $\mathbf{H}_{0}\left(\operatorname{div} 0 ; \mathcal{I}^{3}\right)$ is infinite.

Because of the aforementioned constant extension of functions of $\ell$ variables to a functions of $n$ variables, the divergence-free wavelets that are obtained do not satisfy boundary conditions beyond having vanishing normals. Indeed, one verifies that, e.g., the tangential components at $x_{3}=\{0\}$ or $x_{3}=\{1\}$ of the divergence-free wavelets on $\mathcal{I}^{3}$ that stem from the second subspace at the right-hand side of (1.10) span the whole of $\mathbf{H}_{0}\left(\operatorname{div} 0 ; \mathcal{I}^{2}\right)$. In other words, the construction from [25] is restricted to slip boundary conditions.

The aim of the current paper is to extend the approach to general boundary conditions, including no-slip boundary conditions. The key to achieving this will be the replacement of the orthogonal decomposition $L_{2}(\mathcal{I})=L_{2,0}(\mathcal{I}) \oplus^{\perp} \llbracket \mathbb{1} \rrbracket$ by a biorthogonal decomposition

$$
L_{2}(\mathcal{I})=\left(L_{2}(\mathcal{I}) \cap \llbracket \tilde{\sigma} \rrbracket^{\perp}\right) \oplus \llbracket \sigma \rrbracket, \quad L_{2}(\mathcal{I})=\left(L_{2}(\mathcal{I}) \cap \llbracket \sigma \rrbracket^{\perp}\right) \oplus \llbracket \tilde{\sigma} \rrbracket,
$$

with $\sigma$, $\tilde{\sigma}$ being some functions on $\mathcal{I}$ with $\int_{\mathcal{I}} \sigma \tilde{\sigma} \neq 0$. Any biorthogonal (wavelet) basis for $\left(L_{2}(\mathcal{I}), L_{2}(\mathcal{I})\right)$ gives rise to such a decomposition by identifying one pair of a primal and a dual wavelet as the pair $(\sigma, \tilde{\sigma})$. Starting from this biorthogonal decomposition of $\left(L_{2}(\mathcal{I}), L_{2}(\mathcal{I})\right.$ ), we will construct a biorthogonal decomposition of $\left(L_{2}\left(\mathcal{I}^{n}\right)^{n}, L_{2}\left(\mathcal{I}^{n}\right)^{n}\right)$, with both instances of $L_{2}\left(\mathcal{I}^{n}\right)^{n}$ being split into $2^{n}-1$ subspaces. For $n=3$, this decomposition reads as (1.10) with, at the primal or dual side, $L_{2,0}$ being replaced by $L_{2}(\mathcal{I}) \cap \llbracket \tilde{\sigma} \rrbracket^{\perp}$ or $L_{2}(\mathcal{I}) \cap \llbracket \sigma \rrbracket^{\perp}$, and $\llbracket \mathbb{1} \rrbracket$ by $\llbracket \sigma \rrbracket$ or $\llbracket \tilde{\sigma} \rrbracket$, respectively. The pair $(\sigma, \tilde{\sigma})$ may be chosen differently for each coordinate direction.

As we will see, in view of constructing divergence-free wavelets, the role of $\tilde{\sigma}$ has to be played by the function $\mathbb{1}$, because of its special property of having a zero derivative. Since the sole condition on $\sigma$ (or $\sigma_{i}$ if it depends on the coordinate direction $1 \leq i \leq n$ ) is that $\int_{\mathcal{I}} \sigma \mathbb{1} \neq 0$, the univariate primal wavelets, so in particular $\sigma$, can be arranged to vanish at $\{0\}$ and $\{1\}$ to any given orders. As a consequence, we will be able to construct a wavelet Riesz basis for $\mathbf{H}_{0}\left(\operatorname{div} 0 ; \mathcal{I}^{n}\right)$, that for given $k$, renormalized, will be a basis for $\stackrel{\mathrm{H}}{k}^{k}\left(\mathcal{I}^{n}\right) \cap \mathbf{H}_{0}\left(\operatorname{div} 0 ; \mathcal{I}^{n}\right)$, with the first space being the closed subspace of $H^{k}\left(\mathcal{I}^{n}\right)^{n}$ defined by imposing (very) general homogeneous Dirichlet boundary conditions up to order $k$.

\subsection{Organization of this Paper}

This paper is organized as follows: In the next short Sect. 2, we will recall that the construction of divergence-free wavelet bases in two space dimensions is straightforward 
due to the special properties of the curl operator. This holds true for simply connected Lipschitz domains $\Omega$ as long as one is not interested in properties of a corresponding dual basis. It suffices to have available a wavelet Riesz basis for $H_{0}^{1}(\Omega)$, which is known to be possible on arbitrary polygons.

Section 3 is devoted to the construction of the aforementioned biorthogonal space decomposition of $\left(L_{2}\left(\mathcal{I}^{n}\right)^{n}, L_{2}\left(\mathcal{I}^{n}\right)^{n}\right)$ with both instances of $L_{2}\left(\mathcal{I}^{n}\right)^{n}$ being split into $2^{n}-1$ subspaces. A general framework is presented to construct a divergence-free wavelet basis from divergence-free wavelet bases for the subspaces in the primal decomposition, as well as a dual basis from dual bases inside the corresponding subspaces in the dual decomposition.

To find such divergence-free wavelet bases for the primal subspaces, as well as dual bases in the corresponding dual subspaces, as in [21], we need two pairs of univariate biorthogonal wavelet Riesz bases on $\mathcal{I}$, possibly different in the different coordinate directions, that are related by integration or differentiation as in (1.1). In Sect. 4.1, we will show that given one pair of biorthogonal wavelet Riesz bases, which plays the role of $(\Psi, \tilde{\Psi})$ in $(1.1)$, the related pair, being $(\Psi, \tilde{\Psi})$ in $(1.1)$, can always be constructed. Apart from being Riesz bases for the relevant Sobolev spaces, the sole condition on the first pair is that $\mathbb{1}$ is a dual wavelet, and, when one aims at locally supported divergence-free primal and/or dual wavelets, that the primal and/or dual wavelets of the first pair are locally supported. The findings in this subsection generalize those from [14], where such pairs of biorthogonal wavelet bases are constructed by adapting translation invariant wavelet bases on $\mathbb{R}$ to the interval.

In combination with the results from Sect. 3, in Sect. 4.2, we will end up with wavelet Riesz bases for $\stackrel{\circ}{\mathbf{H}}^{k}\left(\mathcal{I}^{n}\right) \cap \mathbf{H}_{0}\left(\operatorname{div} 0 ; \mathcal{I}^{n}\right)$, as well as with dual bases. The multivariate wavelets will be anisotropic, i.e., tensor products of univariate wavelets.

Finally, in Sect. 5, single-scale bases will be constructed for the spans of the various univariate primal or dual wavelets up to a given level. Using these single-scale bases, we will also construct an isotropic wavelet Riesz bases for $\stackrel{\mathrm{H}}{ }^{k}\left(\mathcal{I}^{2}\right) \cap \mathbf{H}_{0}\left(\operatorname{div} 0 ; \mathcal{I}^{2}\right)$, as well as a dual basis. Other than on $\mathbb{R}^{n}$, we could not manage to do this on $\mathcal{I}^{n}$ for $n \geq 3$. Fortunately, anisotropic approximation has the advantage anyway that the best possible convergence rate does not deteriorate with increasing $n$.

In this work, by $C \lesssim D$, we will mean that $C$ can be bounded by a multiple of $D$, independently of parameters on which $\mathrm{C}$ and $\mathrm{D}$ may depend. Obviously, $C \gtrsim D$ is defined as $D \lesssim C$, and $C \approx D$ as $C \lesssim D$ and $C \gtrsim D$.

\section{Divergence-Free Wavelets in Two Dimensions}

In this short section, we will recall that on two-dimensional domains, the construction of Riesz bases of divergence-free wavelets is rather straightforward because of the special properties of the curl operator in two dimensions.

For $\Omega \subset \mathbb{R}^{2}$ being simply connected with a Lipschitz continuous boundary, it is known that

$$
\text { curl: } H_{0}^{1}(\Omega) \rightarrow \mathbf{H}_{0}(\operatorname{div} 0 ; \Omega) \text { is boundedly invertible. }
$$


Indeed, it is clear that for $v \in H_{0}^{1}(\Omega)$, curl $v \in \mathbf{H}_{0}(\operatorname{div} 0 ; \Omega)$ and $\|\operatorname{curl} v\|_{L_{2}(\Omega)^{2}}=$ $|v|_{H^{1}(\Omega)} \approx\|v\|_{H^{1}(\Omega)}$ by Friedrich's inequality. The remaining nontrivial part is to show that curl is surjective, which is demonstrated in [11, § I.3.1].

As a consequence, for $k \in \mathbb{N}_{>0}$ and a measurable $\Gamma \subset \partial \Omega$, we have that

curl : $H_{0}^{1}(\Omega) \cap H_{0, \Gamma}^{k+1}(\Omega) \rightarrow \mathbf{H}_{0}(\operatorname{div} 0 ; \Omega) \cap H_{0, \Gamma}^{k}(\Omega)^{2}$ is boundedly invertible,

where $H_{0, \Gamma}^{\ell}(\Omega):=\operatorname{clos}_{H^{\ell}(\Omega)}\left\{u \in C(\bar{\Omega}) \cap H^{\ell}(\Omega): u=0\right.$ on $\left.\Gamma\right\}$. Indeed, for $v \in$ $H_{0}^{1}(\Omega) \cap H_{0, \Gamma}^{k+1}(\Omega)$, we have curl $v \in \mathbf{H}_{0}(\operatorname{div} 0 ; \Omega) \cap H_{0, \Gamma}^{k}(\Omega)^{2}$, and $\|\operatorname{curl} v\|_{H^{k}(\Omega)^{2}}^{2}=$ $\sum_{1 \leq|\alpha| \leq k+1}\left\|\partial^{\alpha} v\right\|_{L_{2}(\Omega)}^{2} \approx\|v\|_{H^{k+1}(\Omega)}^{2}$ by Friedrich's inequality. To show surjectivity, given $\mathbf{w} \in \mathbf{H}_{0}(\operatorname{div} 0 ; \Omega) \cap H_{0, \Gamma}^{k}(\Omega)^{2}$, let $v \in H_{0}^{1}(\Omega)$ be such that $\operatorname{curl} v=\mathbf{w}$. Then $v \in H^{k+1}(\Omega)$, and for $1 \leq|\alpha| \leq k+1$, we have that $\partial^{\alpha} v$ vanishes at $\Gamma$, or $v \in$ $H_{0}^{1}(\Omega) \cap H_{0, \Gamma}^{k+1}(\Omega)$.

From (2.2), we conclude that if $\Sigma$ is a Riesz basis for $H_{0}^{1}(\Omega) \cap H_{0, \Gamma}^{k+1}(\Omega)$, e.g., of wavelet type, then $\boldsymbol{\Psi}:=\operatorname{curl} \Sigma$ is a Riesz basis for $\mathbf{H}_{0}(\operatorname{div} 0 ; \Omega) \cap H_{0, \Gamma}^{k}(\Omega)^{2}$.

With the approach of constructing a basis of divergence-free wavelets discussed so far, there is no guarantee that a dual basis consisting of locally supported functions exists. For $\Omega=\mathcal{I}^{2}$, in the next sections, starting from biorthogonal univariate wavelet bases, we will construct both anisotropic and isotropic divergence-free wavelet Riesz bases, with duals that are locally supported whenever the univariate duals have this property. The results for the anisotropic wavelets will be valid for $\Omega=\mathcal{I}^{n}$ for arbitrary dimension $n$, being the main point of this work.

\section{A Biorthogonal Space Decomposition of $\left(L_{2}\left(\mathcal{I}^{n}\right)^{n}, L_{2}\left(\mathcal{I}^{n}\right)^{n}\right)$}

In this section, we split $\left(L_{2}\left(\mathcal{I}^{n}\right)^{n}, L_{2}\left(\mathcal{I}^{n}\right)^{n}\right)$ into $2^{n}-1$ pairs of subspaces such that the union of Riesz bases for the divergence-free parts of the primal subspaces is a Riesz basis for $\mathbf{H}_{0}\left(\operatorname{div} 0 ; \mathcal{I}^{n}\right)$. As we will see in the next section, such bases for the subspaces can be constructed following the approach from [21].

Definition 3.1 For $\emptyset \neq S=\left\{j_{1}, \ldots, j_{\# S}\right\} \subset\{1, \ldots, n\}$, let $L_{2}\left(\mathcal{I}^{S}\right)$ be the space of functions of $\left(x_{j_{1}}, \ldots, x_{j \# S}\right)$ that are square integrable over $\mathcal{I}^{\# S}$. With $L_{2}\left(\mathcal{I}^{S}\right)^{S}$, we will denote the space of $\mathbf{v}=\left(v_{j_{1}}, \ldots, v_{j_{\# S}}\right)$ for which $v_{j_{i}} \in L_{2}\left(\mathcal{I}^{S}\right)(\forall i)$. Analogous definitions will be used for $H^{k}\left(\mathcal{I}^{S}\right)$ and $H^{k}\left(\mathcal{I}^{S}\right)^{S}$. Note that $L_{2}\left(\mathcal{I}^{\{1, \ldots, n\}}\right)=L_{2}\left(\mathcal{I}^{n}\right)$ and $L_{2}\left(\mathcal{I}^{\{1, \ldots, n\}}\right)^{\{1, \ldots, n\}}=L_{2}\left(\mathcal{I}^{n}\right)^{n}$.

For each $1 \leq i \leq n$, we fix two functions $\sigma_{i}, \tilde{\sigma}_{i} \in L_{2}(\mathcal{I})$ with $\left\langle\sigma_{i}, \tilde{\sigma}_{i}\right\rangle_{L_{2}(\mathcal{I})}=1$. We set the biorthogonal projectors $P_{i}, \tilde{P}_{i}$ by

$$
P_{i} u:=\left\langle u, \tilde{\sigma}_{i}\right\rangle_{L_{2}(\mathcal{I})} \sigma_{i}, \quad \tilde{P}_{i} u:=\left\langle u, \sigma_{i}\right\rangle_{L_{2}(\mathcal{I})} \tilde{\sigma}_{i}
$$


For any $\emptyset \neq S=\left\{j_{1}, \ldots, j_{\# S}\right\} \subset\{1, \ldots, n\}$, we set

$$
\begin{aligned}
& \mathbf{L}_{2,0}\left(\mathcal{I}^{S}\right) \\
& \quad:=\left\{\mathbf{v} \in L_{2}\left(\mathcal{I}^{S}\right)^{S}: \int_{\mathcal{I}} v_{j_{i}}\left(x_{j_{1}}, \ldots, x_{j_{\# S}}\right) \tilde{\sigma}_{j_{k}}\left(x_{j_{k}}\right) d x_{j_{k}}=0(1 \leq i \neq k \leq \# S)\right\},
\end{aligned}
$$

and $\mathbf{L}_{2,0}\left(\mathcal{I}^{n}\right):=\mathbf{L}_{2,0}\left(\mathcal{I}^{\{1, \ldots, n\}}\right)$.

At the dual side, we get an analogous definition of $\tilde{\mathbf{L}}_{2,0}\left(\mathcal{I}^{S}\right)$ by replacing $\tilde{\sigma}_{j_{k}}$ by $\sigma_{j_{k}}$.

On many occasions, we will impose that $\tilde{\sigma}_{i} \in \operatorname{span}\{\mathbb{1}\}(\forall i)$. Then $\mathbf{L}_{2,0}\left(\mathcal{I}^{S}\right)$ is the space of $\left(v_{j_{1}}, \ldots, v_{j_{\# S}}\right) \in L_{2}(S)^{S}$, where, for any $k \neq i, v_{j_{i}}$ has zero mean as a function of $x_{j_{k}}$ when frozen in the other variables. So, in particular, in this case the definition of $\mathbf{L}_{2,0}\left(\mathcal{I}^{n}\right)$ coincides with the one given earlier in the introduction in (1.2).

Next, we will construct an isomorphism between $\prod_{\emptyset \neq S \subset\{1, \ldots, n\}} \mathbf{L}_{2,0}\left(\mathcal{I}^{S}\right)$, and similarly $\prod_{\emptyset \neq S \subset\{1, \ldots, n\}} \tilde{\mathbf{L}}_{2,0}\left(\mathcal{I}^{S}\right)$, and $L_{2}\left(\mathcal{I}^{n}\right)^{n}$.

Definition 3.2 We define embeddings and projectors

$$
E^{(S)}: \mathbf{L}_{2,0}\left(\mathcal{I}^{S}\right) \rightarrow L_{2}\left(\mathcal{I}^{n}\right)^{n}, \quad Q^{(S)}: L_{2}\left(\mathcal{I}^{n}\right)^{n} \rightarrow \mathbf{L}_{2,0}\left(\mathcal{I}^{S}\right)
$$

by

$$
\left(E^{(S)} \mathbf{v}\right)(x):=\sum_{i=1}^{\# S}\left[v_{j_{i}}\left(x_{j_{1}}, \ldots, x_{j_{\# S}}\right) \prod_{k \in\{1, \ldots, n\} \backslash S} \sigma_{k}\left(x_{k}\right)\right] \mathbf{e}_{j_{i}},
$$

where $\mathbf{e}_{s_{i}}$ denotes the $s_{i}$ th canonical unit vector in $\mathbb{R}^{n}$, and

$$
\left(Q^{(S)} \mathbf{v}\right)_{i}:=\frac{\int_{\mathcal{I}^{n-\# S}} Q_{i 1}^{(S)} \otimes \cdots \otimes Q_{i n}^{(S)} v_{i} \mathrm{~d} x_{k_{1}} \ldots \mathrm{d} x_{k_{n-\# S}}}{\prod_{k \in\{1, \ldots, n\} \backslash S} \int_{\mathcal{I}} \sigma_{k}} \quad(i \in S),
$$

where $\left\{k_{1}, \ldots, k_{n-\# S}\right\}:=\{1, \ldots, n\} \backslash S$, and $Q_{i j}^{(S)}:=\left\{\begin{array}{cc}I & i=j \\ I-P_{j} & i \neq j \\ P_{j} & j \notin S\end{array}\right\}$.

At the dual side, we get an analogous definition of $\tilde{E}^{(S)}$ and $\tilde{Q}^{(S)}$ by replacing $\left(\mathbf{L}_{2,0}\left(\mathcal{I}^{S}\right), \sigma_{k}, P_{j}\right)$ by $\left(\tilde{\mathbf{L}}_{2,0}\left(\mathcal{I}^{S}\right), \tilde{\sigma}_{k}, \tilde{P}_{j}\right)$.

Note that when $\tilde{\sigma}_{i} \in \operatorname{span}\{\mathbb{1}\}(\forall i)$, then $E^{(S)}$ is the embedding that was discussed in Sect. 1.4 below (1.10). It extends the nonzero coordinate functions $v_{j_{1}}, \ldots, v_{j_{\#} S}$ as constant functions of the variables $x_{i}$ for $i \notin\left\{j_{1}, \ldots, j_{\# S}\right\}$. 
Proposition 3.3 The bounded mappings

$$
\begin{aligned}
& E: \prod_{\emptyset \neq S \subset\{1, \ldots, n\}} \mathbf{L}_{2,0}\left(\mathcal{I}^{S}\right) \rightarrow L_{2}\left(\mathcal{I}^{n}\right)^{n}:\left(\mathbf{v}^{(S)}\right)_{S} \mapsto \sum_{S} E^{(S)} \mathbf{v}^{(S)}, \\
& Q: L_{2}\left(\mathcal{I}^{n}\right)^{n} \rightarrow \prod_{\emptyset \neq S \subset\{1, \ldots, n\}} \mathbf{L}_{2,0}\left(\mathcal{I}^{S}\right): \mathbf{v} \mapsto\left(Q^{(S)} \mathbf{v}\right)_{S}
\end{aligned}
$$

are each other's inverse.

Defining $\tilde{E}$, $\tilde{Q}$ similarly by replacing $\left(\mathbf{L}_{2,0}\left(\mathcal{I}^{S}\right), E^{(S)}, Q^{(S)}\right)$ by $\left(\tilde{\mathbf{L}}_{2,0}\left(\mathcal{I}^{S}\right), \tilde{E}^{(S)}, \tilde{Q}^{(S)}\right)$, the analogous result is valid at the dual side.

Proof For $\emptyset \neq S, S^{\prime} \subset\{1, \ldots, n\}$, consider $\left(Q^{\left(S^{\prime}\right)} E^{(S)} \mathbf{w}\right)_{i}$ for $i \in S^{\prime}$. If $i \notin S$, then $(\cdots)_{i}=0$ since $\left(E^{(S)} \mathbf{w}\right)_{i}=0$. If $i \in S$ and $\exists S^{\prime} \ni j \neq i$ with $j \notin S$, then $(\cdots)_{i}=0$ since $\left(I-P_{j}\right) \sigma_{j}=0$. If $i \in S$ and $S \ni j \neq i$ with $j \notin S^{\prime}$, then $(\cdots)_{i}=0$ since $P_{j} u=0$ when $u \perp_{L_{2}(\mathcal{I})} \operatorname{span}\left\{\tilde{\sigma}_{j}\right\}$. So $Q^{\left(S^{\prime}\right)} E^{(S)}=0$ when $S^{\prime} \neq S$. Because of $P_{j} \sigma_{j}=\sigma_{j}$, and $\left(I-P_{j}\right) u=u$ when $u \perp_{L_{2}(\mathcal{I})} \operatorname{span}\left\{\tilde{\sigma}_{j}\right\}$, similarly we infer $Q^{(S)} E^{(S)}=I$. We conclude that $Q E=I$.

From $\sum_{\emptyset \neq S \subset\{1, \ldots, n\}}\left(E^{(S)} Q^{(S)} v\right)_{i}=\sum_{\{1, \ldots, n\} \supset S \ni\{i\}} Q_{i 1}^{(S)} \otimes \cdots \otimes Q_{i n}^{(S)} v_{i}=v_{i}$, we have $E Q=I$.

As an easy consequence of Proposition 3.3, we have the space decompositions

$$
L_{2}\left(\mathcal{I}^{n}\right)^{n}=\bigoplus_{\emptyset \neq S \subset\{1, \ldots, n\}} \operatorname{ran} E^{(S)}, \quad L_{2}\left(\mathcal{I}^{n}\right)^{n}=\bigoplus_{\emptyset \neq S \subset\{1, \ldots, n\}} \operatorname{ran} \tilde{E}^{(S)}
$$

Example 3.4 Recalling the abbreviations $L_{2}:=L_{2}(\mathcal{I})$ and $\llbracket \cdot \rrbracket:=\operatorname{span}\{\cdot\}$, and setting

$$
L_{2}^{(i)}:=L_{2} \cap \llbracket \tilde{\sigma}_{i} \rrbracket^{\perp},
$$

the decomposition $L_{2}\left(\mathcal{I}^{n}\right)^{n}=\bigoplus_{\emptyset \neq S \subset\{1, \ldots, n\}}$ ran $E^{(S)}$ for the cases $n=2$ and $n=3$ (the most relevant case) reads as follows:

$$
\begin{aligned}
L_{2}\left(\mathcal{I}^{2}\right)^{2}= & L_{2} \otimes L_{2}^{(2)} \times L_{2}^{(1)} \otimes L_{2} & & (S=\{1,2\}) \\
\oplus L_{2} \otimes \llbracket \sigma_{2} \rrbracket \times & \times & & (S=\{1\}) \\
\oplus \quad 0 & \times \llbracket \sigma_{1} \rrbracket \otimes L_{2} & & (S=\{2\}),
\end{aligned}
$$

and

$$
\begin{aligned}
& L_{2}\left(\mathcal{I}^{3}\right)^{3}=L_{2} \otimes L_{2}^{(2)} \otimes L_{2}^{(3)} \times L_{2}^{(1)} \otimes L_{2} \otimes L_{2}^{(3)} \times L_{2}^{(1)} \otimes L_{2}^{(2)} \otimes L_{2} \quad(S=\{1,2,3\}) \\
& \oplus L_{2} \otimes L_{2}^{(2)} \otimes \llbracket \sigma_{3} \rrbracket \times L_{2}^{(1)} \otimes L_{2} \otimes \llbracket \sigma_{3} \rrbracket \times \quad 0 \quad(S=\{1,2\}) \\
& \oplus L_{2} \otimes \llbracket \sigma_{2} \rrbracket \otimes L_{2}^{(3)} \times \quad 0 \quad \times L_{2}^{(1)} \otimes \llbracket \sigma_{2} \rrbracket \otimes L_{2} \quad(S=\{1,3\}) \\
& \oplus \quad 0 \quad \times \llbracket \sigma_{1} \rrbracket \otimes L_{2} \otimes L_{2}^{(3)} \times \llbracket \sigma_{1} \rrbracket \otimes L_{2}^{(2)} \otimes L_{2} \quad(S=\{2,3\}) \\
& \oplus L_{2} \otimes \llbracket \sigma_{2} \rrbracket \otimes \llbracket \sigma_{3} \rrbracket \times \quad 0 \quad \times \quad 0 \quad(S=\{1\}) \\
& \oplus \quad 0 \quad \times \llbracket \sigma_{1} \rrbracket \otimes L_{2} \otimes \llbracket \sigma_{3} \rrbracket \times \quad 0 \quad(S=\{2\}) \\
& \oplus \quad 0 \quad \times \quad 0 \quad \times \llbracket \sigma_{1} \rrbracket \otimes \llbracket \sigma_{3} \rrbracket \otimes L_{2} \quad(S=\{3\}) .
\end{aligned}
$$


The decompositions at the dual side are obtained by replacing $\sigma_{i}$ by $\tilde{\sigma}_{i}$ and $L_{2}^{(i)}$ by $\tilde{L}_{2}^{(i)}:=L_{2} \cap \llbracket \sigma_{i} \rrbracket^{\perp}$.

As shown in the next proposition, the decompositions in (3.1) are biorthogonal.

Proposition 3.5 For $\emptyset \neq S, S^{\prime} \subset\{1, \ldots, n\}$, we have $\left(Q^{(S)}\right)^{*}=\tilde{E}^{(S)},\left(\tilde{Q}^{(S)}\right)^{*}=$ $E^{(S)}$, and $\left(\tilde{E}^{\left(S^{\prime}\right)}\right)^{*} E^{(S)}=\left\{\begin{array}{ll}I & S=S^{\prime} \\ 0 & S \neq S^{\prime}\end{array}\right\}$.

Proof To show the first, and so the similar second statement, it is sufficient to consider $S=\{1, \ldots, \ell\}$ for some $1 \leq \ell \leq n$. For $\mathbf{v} \in L_{2}\left(\mathcal{I}^{n}\right)^{n}$ and $\mathbf{w} \in \tilde{\mathbf{L}}_{2,0}\left(\mathcal{I}^{S}\right)$, it is sufficient to verify whether for $1 \leq \bar{i} \leq \ell,\left\langle\left(Q^{(S)} \mathbf{v}\right)_{i}, w_{i}\right\rangle_{L_{2}\left(\mathcal{I}^{\ell}\right)}=\left\langle v_{i},\left(\tilde{E}^{(S)} \mathbf{w}\right)_{i}\right\rangle_{L_{2}\left(\mathcal{I}^{n}\right)}$. Note that $\left(Q^{(S)} \mathbf{v}\right)_{i},\left(\tilde{E}^{(S)} \mathbf{w}\right)_{i}$ depend only on $v_{i}, w_{i}$, respectively. W.l.o.g. let $i=1$. It is sufficient to consider $v_{1}=\otimes_{i=1}^{n} r_{i}, w_{1}=\otimes_{i=1}^{\ell} s_{i}$, where $r_{i}, s_{i} \in L_{2}(\mathcal{I})$ with, for $i \geq 2,\left\langle s_{i}, \sigma_{i}\right\rangle_{L_{2}(\mathcal{I})}=0$. Now from

$$
\begin{aligned}
& \left(Q^{(S)} \mathbf{v}\right)_{1}=\left(\prod_{i=\ell+1}^{n}\left\langle r_{i}, \tilde{\sigma}_{i}\right\rangle_{L_{2}(\mathcal{I})}\right) r_{1} \otimes\left(I-P_{2}\right) r_{2} \otimes \cdots \otimes\left(I-P_{\ell}\right) r_{\ell}, \\
& \left(\tilde{E}^{(S)} \mathbf{w}\right)_{1}=s_{1} \otimes \cdots \otimes s_{\ell} \otimes \tilde{\sigma}_{\ell+1} \otimes \cdots \otimes \tilde{\sigma}_{n},
\end{aligned}
$$

the first, and so second statement follow.

The last statement follows from the first one using that $Q E=I$.

Next, we define Sobolev spaces of divergence-free functions.

Definition 3.6 For $\emptyset \neq S \subset\{1, \ldots, n\}$, on $H^{1}\left(\mathcal{I}^{S}\right)$ we set $\operatorname{grad} v=\left(\partial_{x_{j_{1}}} v, \ldots\right.$,

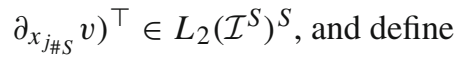

$$
\mathbf{H}_{0}\left(\operatorname{div} 0 ; \mathcal{I}^{S}\right)=\left\{\mathbf{v} \in L_{2}\left(\mathcal{I}^{S}\right)^{S}: \sum_{i \in S} \partial_{x_{i}} v_{i}=0,\left.v_{i}\right|_{x_{i} \in\{0,1\}}=0(i \in S)\right\}
$$

Note that $\mathbf{H}_{0}\left(\operatorname{div} 0 ; \mathcal{I}^{S}\right)=\{0\}$ when $\# S=1$, and that

$\mathbf{H}_{0}\left(\operatorname{div} 0 ; \mathcal{I}^{n}\right):=\mathbf{H}_{0}\left(\operatorname{div} 0 ; \mathcal{I}^{\{1, \ldots, n\}}\right)=\left\{\mathbf{v} \in L_{2}\left(\mathcal{I}^{n}\right): \operatorname{div} \mathbf{v}=0, \mathbf{v} \cdot \mathbf{n}=0\right.$ on $\left.\partial \mathcal{I}^{n}\right\}$

Furthermore, recall the Helmholtz decomposition

$$
L_{2}\left(\mathcal{I}^{S}\right)^{S}=\mathbf{H}_{0}\left(\operatorname{div} 0 ; \mathcal{I}^{S}\right) \oplus^{\perp} \operatorname{grad} H^{1}\left(\mathcal{I}^{S}\right)
$$

It is an easy consequence of the fact that for $\mathbf{u} \in L_{2}\left(\mathcal{I}^{S}\right)^{S}$, the solution $v \in \bar{H}^{1}\left(\mathcal{I}^{S}\right):=$ $\left\{w \in H^{1}\left(\mathcal{I}^{S}\right): \int_{\mathcal{I}^{S}} w=0\right\}$ of $\int_{\mathcal{I}^{S}} \operatorname{grad} v \cdot \operatorname{grad} w=\int_{\mathcal{I}^{S}} \mathbf{u} \cdot \operatorname{grad} w\left(w \in \bar{H}^{1}\left(\mathcal{I}^{S}\right)\right)$ satisfies $\mathbf{u}-\operatorname{grad} v \in \mathbf{H}_{0}\left(\operatorname{div} 0 ; \mathcal{I}^{S}\right)$. 
Proposition 3.7 For $1 \leq i \leq n$, let $0 \neq \tilde{\sigma}_{i} \in \operatorname{span}\{\mathbb{1}\}$. Then for $\emptyset \neq S \subset\{1, \ldots, n\}$,

$$
\begin{aligned}
\tilde{E}^{(S)}\left(\tilde{\mathbf{L}}_{2,0}\left(\mathcal{I}^{S}\right) \cap \operatorname{grad} H^{1}\left(\mathcal{I}^{S}\right)\right) & \subset \operatorname{grad} H^{1}\left(\mathcal{I}^{n}\right), \\
E^{(S)}\left(\mathbf{L}_{2,0}\left(\mathcal{I}^{S}\right) \cap \mathbf{H}_{0}\left(\operatorname{div} 0 ; \mathcal{I}^{S}\right)\right) & \subset \mathbf{H}_{0}\left(\operatorname{div} 0 ; \mathcal{I}^{n}\right), \\
Q^{(S)}\left(\mathbf{H}_{0}\left(\operatorname{div} 0 ; \mathcal{I}^{n}\right)\right) & \subset \mathbf{L}_{2,0}\left(\mathcal{I}^{S}\right) \cap \mathbf{H}_{0}\left(\operatorname{div} 0 ; \mathcal{I}^{S}\right), \\
\tilde{Q}^{(S)}\left(\operatorname{grad} H^{1}\left(\mathcal{I}^{n}\right)\right) & \subset \tilde{\mathbf{L}}_{2,0}\left(\mathcal{I}^{S}\right) \cap \operatorname{grad} H^{1}\left(\mathcal{I}^{S}\right) .
\end{aligned}
$$

Proof The first two results follow directly from the definitions of $\tilde{E}^{(S)}$ and $E^{(S)}$. For the first one, it is used that the $\tilde{\sigma}_{i}$ 's are multiples of $\mathbb{1}$.

For $\mathbf{u} \in \mathbf{H}_{0}\left(\operatorname{div} 0 ; \mathcal{I}^{n}\right), v \in L_{2}\left(\mathcal{I}^{n}\right)$, Proposition 3.5, (3.2), and the first statement show that

$$
\left\langle Q^{(S)} \mathbf{u}, \nabla v\right\rangle_{L_{2}\left(\mathcal{I}^{S}\right)^{S}}=\left\langle\mathbf{u}, \tilde{E}^{(S)} \nabla v\right\rangle_{L_{2}\left(\mathcal{I}^{n}\right)^{n}}=0,
$$

which shows the third statement again by (3.2). The last statement is proved analogously.

For $\emptyset \neq S \subset\{1, \ldots, n\}$, we define Sobolev spaces, being subspaces of $L_{2}(S)^{S}$, of vector fields whose coordinates satisfy homogeneous Dirichlet boundary conditions of certain orders. Since we are working on the hypercube, these boundary conditions can be identified as normal or tangential boundary conditions on the vector field. For any $1 \leq i \leq n$ and $b \in\{0,1\}$, we will fix two integer parameters $n_{b}^{(i)}$ and $t_{b}^{(i)}$. They will be the orders of the normal or of all tangential boundary conditions at $x_{i}=b$, respectively, when $i \in S$. So the orders of the boundary conditions in different Cartesian tangential directions at $x_{i}=b$ cannot be chosen individually. Although the Sobolev spaces depend on these $4 n$ parameters, this will not be expressed in their notation, to avoid making them too heavy.

Definition 3.8 Let $k \in \mathbb{N}:=\{0,1, \ldots\}$. Fixing, for $1 \leq i \leq n$ and $b \in\{0,1\}$, $n_{b}^{(i)}, t_{b}^{(i)} \in\{0, \ldots, k\}$, for $\emptyset \neq S \subset\{1, \ldots, n\}$, let

$$
\begin{array}{r}
\stackrel{\circ}{\mathbf{H}}^{k}\left(\mathcal{I}^{S}\right)=\left\{\mathbf{v} \in H^{k}\left(\mathcal{I}^{S}\right)^{S}:\left.\partial_{x_{i}}^{p} v_{i}\right|_{x_{i}=b}=0\left(i \in S, 0 \leq p \leq n_{b}^{(i)}-1, b \in\{0,1\}\right),\right. \\
\left.\left.\partial_{x_{j}}^{p} v_{i}\right|_{x_{j}=b}=0\left(i \neq j \in S, 0 \leq p \leq t_{b}^{(j)}-1, b \in\{0,1\}\right)\right\},
\end{array}
$$

and $\stackrel{\circ}{\mathbf{H}}^{k}\left(\mathcal{I}^{n}\right)=\stackrel{\circ}{\mathbf{H}}^{k}\left(\mathcal{I}^{\{1, \ldots, n\}}\right)$.

For $p_{0}, p_{1} \in\{0, \ldots, k\}$, we set

$$
H_{\left(p_{0}, p_{1}\right)}^{k}(\mathcal{I})=\left\{u \in H^{k}(\mathcal{I}): u^{(p)}(b)=0\left(0 \leq p \leq p_{b}-1, b \in\{0,1\}\right)\right\} .
$$

Note that $H_{(0,0)}^{k}(\mathcal{I})=H^{k}(\mathcal{I})$ and $H_{(k, k)}^{k}(\mathcal{I})=H_{0}^{k}(\mathcal{I})$.

Remark 3.9 Although for ease of presentation, we consider only Sobolev spaces with integer orders, everything can be generalized to noninteger orders, with some care for orders in $\mathbb{N}+\frac{1}{2}$. 
The following proposition extends upon Proposition 3.3.

Proposition 3.10 For $1 \leq i \leq n$, let $\sigma_{i} \in H_{\left(t_{0}^{(i)}, t_{1}^{(i)}\right)}^{k}(\mathcal{I})$. Then

$E: \prod_{\emptyset \neq S \subset\{1, \ldots, n\}} \mathbf{L}_{2,0}\left(\mathcal{I}^{S}\right) \cap \stackrel{\circ}{\mathbf{H}}^{k}\left(\mathcal{I}^{S}\right) \rightarrow \stackrel{\circ}{\mathbf{H}}^{k}\left(\mathcal{I}^{n}\right)$ is boundedly invertible.

Proof Thanks to the condition on the $\sigma_{i}, E^{(S)}: \mathbf{L}_{2,0}\left(\mathcal{I}^{S}\right) \cap \stackrel{\circ}{\mathbf{H}}^{k}\left(\mathcal{I}^{S}\right) \rightarrow \stackrel{\circ}{\mathbf{H}}^{k}\left(\mathcal{I}^{n}\right)$ and $Q^{(S)}: \stackrel{\circ}{\mathbf{H}}^{k}\left(\mathcal{I}^{n}\right) \rightarrow \mathbf{L}_{2,0}\left(\mathcal{I}^{S}\right) \cap \stackrel{\circ}{\mathbf{H}}^{k}\left(\mathcal{I}^{S}\right)$ are bounded. Now use that $E^{-1}=Q$ by Proposition 3.3.

Below, we will assume that $\tilde{\sigma}_{i} \in \operatorname{span}\{\mathbb{1}\}(\forall i)$. Obviously these functions are smooth but do not satisfy homogeneous boundary conditions of any order. Consequently, the corresponding statement of Proposition 3.10 at the dual side reads as

$\tilde{E}: \prod_{\emptyset \neq S \subset\{1, \ldots, n\}} \tilde{\mathbf{L}}_{2,0}\left(\mathcal{I}^{S}\right) \cap H^{k}\left(\mathcal{I}^{S}\right)^{S} \rightarrow H^{k}\left(\mathcal{I}^{n}\right)^{n} \quad$ is boundedly invertible.

As a consequence of Propositions 3.7 and 3.10, we have:

Corollary 3.11 For $1 \leq i \leq n$, let $\sigma_{i} \in H_{\left(t_{0}^{(i)}, t_{1}^{(i)}\right)}^{k}(\mathcal{I})$ and $0 \neq \tilde{\sigma}_{i} \in \operatorname{span}\{\mathbb{1}\}$. Then both

$E: \quad \prod_{\{S \subset\{1, \ldots, n\}: \# S \geq 2\}} \mathbf{L}_{2,0}\left(\mathcal{I}^{S}\right) \cap \stackrel{\circ}{\mathbf{H}}^{k}\left(\mathcal{I}^{S}\right) \cap \mathbf{H}_{0}\left(\operatorname{div} 0 ; \mathcal{I}^{S}\right) \rightarrow \stackrel{\circ}{\mathbf{H}}^{k}\left(\mathcal{I}^{n}\right) \cap \mathbf{H}_{0}\left(\operatorname{div} 0 ; \mathcal{I}^{n}\right)$, $\tilde{E}: \prod_{\emptyset \neq S \subset\{1, \ldots, n\}} \tilde{\mathbf{L}}_{2,0}\left(\mathcal{I}^{S}\right) \cap H^{k}\left(\mathcal{I}^{S}\right)^{S} \cap \operatorname{grad} H^{1}\left(\mathcal{I}^{S}\right) \rightarrow H^{k}\left(\mathcal{I}^{n}\right)^{n} \cap \operatorname{grad} H^{1}\left(\mathcal{I}^{n}\right)$,

are boundedly invertible.

We are ready to formulate one of the two main ingredients for the construction of the divergence-free wavelet basis (the other one is the construction of the collections $\Psi_{\mathrm{df}}^{(S)}$ that is given in the forthcoming Theorem 4.4).

Corollary 3.12 For $1 \leq i \leq n$, let $\sigma_{i} \in H_{\left(t_{0}^{(i)}, t_{1}^{(i)}\right)}^{k}(\mathcal{I})$ and $0 \neq \tilde{\sigma}_{i} \in \operatorname{span}\{\mathbb{1}\}$. For $S \subset$ $\{1, \ldots, n\}, \# S \geq 2$, let $\boldsymbol{\Psi}_{\mathrm{df}}^{(S)}$ be a Riesz basis for $\mathbf{L}_{2,0}\left(\mathcal{I}^{S}\right) \cap \stackrel{\circ}{\mathbf{H}}^{k}\left(\mathcal{I}^{S}\right) \cap \mathbf{H}_{0}\left(\operatorname{div} 0 ; \mathcal{I}^{S}\right)$. Then

$$
\left.\bigcup_{\{S \subset\{1, \ldots, n\}: \# S \geq 2\}} E^{(S)} \Psi_{\mathrm{df}}^{(S)} \text { is a Riesz basis for } \stackrel{\circ}{\mathbf{H}}^{k}\left(\mathcal{I}^{n}\right) \cap \mathbf{H}_{0} \operatorname{div0;} \mathcal{I}^{n}\right) .
$$

If, furthermore, $\tilde{\mathbf{\Psi}}_{\mathrm{df}}^{(S)} \subset \tilde{\mathbf{L}}_{2,0}\left(\mathcal{I}^{S}\right)$ is a dual basis for $\boldsymbol{\Psi}_{\mathrm{df}}^{(S)}$, then a dual basis is given by

$$
\bigcup_{\{S \subset\{1, \ldots, n\}: \# S \geq 2\}} \tilde{E}^{(S)} \tilde{\boldsymbol{\Psi}}_{\mathrm{df}}^{(S)}
$$


Proof The first statement is obvious, and the biorthogonality is a consequence of Proposition 3.5.

The collection $\tilde{\boldsymbol{\Psi}}_{\mathrm{df}}^{(S)}$ being a dual basis additionally means that for $\mathbf{v} \in \mathbf{L}_{2,0}\left(\mathcal{I}^{S}\right) \cap \stackrel{\circ}{\mathbf{H}}^{k}\left(\mathcal{I}^{S}\right),\left\|\left\langle\mathbf{v}, \tilde{\Psi}_{\mathrm{df}}^{(S)}\right\rangle_{L_{2}(S)^{S}}\right\|_{\ell_{2}} \lesssim\|\mathbf{v}\|_{H^{k}\left(\mathcal{I}^{S}\right)^{S}}$. Now let $\mathbf{u} \in \stackrel{\circ}{\mathbf{H}}^{k}\left(\mathcal{I}^{n}\right)^{n}$. Then

$$
\begin{aligned}
& \sum_{\{S \subset\{1, \ldots, n\}: \# S \geq 2\}}\left\|\left\langle\mathbf{u}, \tilde{E}^{(S)} \tilde{\boldsymbol{\Psi}}_{\mathrm{df}}^{(S)}\right\rangle_{L_{2}\left(\mathcal{I}^{n}\right)^{n}}\right\|_{\ell_{2}}^{2}=\sum_{\{S \subset\{1, \ldots, n\}: \# S \geq 2\}}\left\|\left\langle Q^{(S)} \mathbf{u}, \tilde{\boldsymbol{\Psi}}_{\mathrm{df}}^{(S)}\right\rangle_{L_{2}(S)^{S}}\right\|_{\ell_{2}}^{2} \\
& \lesssim \sum_{\{S \subset\{1, \ldots, n\}: \# S \geq 2\}}\left\|Q^{(S)} \mathbf{u}\right\|_{H^{k}\left(\mathcal{I}^{S}\right)^{S}}^{2} \lesssim\|\mathbf{u}\|_{H^{k}\left(\mathcal{I}^{n}\right)^{n}}^{2},
\end{aligned}
$$

which completes the proof of $\bigcup_{\{S \subset\{1, \ldots, n\}: \# S \geq 2\}} \tilde{E}^{(S)} \tilde{\boldsymbol{\Psi}}_{\mathrm{df}}^{(S)}$ being a dual basis.

Obviously, a similar result can be formulated for the construction of a Riesz basis for $H^{k}\left(\mathcal{I}^{n}\right)^{n} \cap \operatorname{grad} H^{1}\left(\mathcal{I}^{n}\right)$.

\section{Construction of Divergence-Free Wavelets on the Subspaces}

For $S \subset\{1, \ldots, n\}, \# S \geq 2$, biorthogonal collections $\left(\boldsymbol{\Psi}_{\mathrm{df}}^{(S)}, \tilde{\boldsymbol{\Psi}}_{\mathrm{df}}^{(S)}\right) \subset \mathbf{L}_{2,0}\left(\mathcal{I}^{S}\right) \times$ $\tilde{\mathbf{L}}_{2,0}\left(\mathcal{I}^{S}\right)$ are constructed as needed in Corollary 3.12. As building blocks, first two pairs of univariate biorthogonal wavelet bases will be constructed, possibly different for each coordinate direction.

\subsection{Pairs of Biorthogonal Riesz Bases on the Interval Related via Differentiation/Integration}

Starting from a general pair of biorthogonal univariate wavelet bases $\left(\Psi^{(i)}, \tilde{\Psi}^{(i)}\right)$ $(1 \leq i \leq n)$ for $\left(L_{2}(\mathcal{I}), L_{2}(\mathcal{I})\right)$, in this subsection a new pair is constructed by integration/differentiation. This construction generalizes the one from [21] for the stationary multiresolution case on the line, as well as those from [14] for stationary multiresolution analyses adapted to the interval.

We will require that the collection of dual wavelets is such that there exists a dual wavelet that is a multiple of $\mathbb{1}$. This condition means that all primal wavelets, except for the one that forms a biorthogonal pair with the multiple of $\mathbb{1}$, have at least one vanishing moment, and that no Dirichlet boundary conditions are incorporated in the dual system. The special biorthogonal pair will play the role of $\left(\sigma_{i}, \tilde{\sigma}_{i}\right)$ in the construction of the biorthogonal space decomposition of $\left(L_{2}\left(\mathcal{I}^{n}\right)^{n}, L_{2}\left(\mathcal{I}^{n}\right)^{n}\right)$ discussed in Sect. 3, and will be excluded from the integration/differentiation process.

Theorem 4.1 For $1 \leq i \leq n, k \in \mathbb{N}$, and $t_{0}^{(i)}, t_{1}^{(i)} \in\{0, \ldots, k\}$, assume that:

(1) $\Psi^{(i)}=\left\{\psi_{\lambda}^{(i)}: \lambda \in \nabla^{(i)}\right\}, \tilde{\Psi}^{(i)}=\left\{\tilde{\psi}_{\lambda}^{(i)}: \lambda \in \nabla^{(i)}\right\}$ are $L_{2}(\mathcal{I})$-biorthogonal collections,

(2) $\exists \hat{\lambda}^{(i)} \in \nabla^{(i)}$ such that for $\left(\sigma_{i}, \tilde{\sigma}_{i}\right):=\left(\psi_{\hat{\lambda}^{(i)}}^{(i)}, \tilde{\psi}_{\hat{\lambda}^{(i)}}^{(i)}\right)$, it holds that $\tilde{\sigma}_{i} \in \operatorname{span}\{\mathbb{1}\}$, 


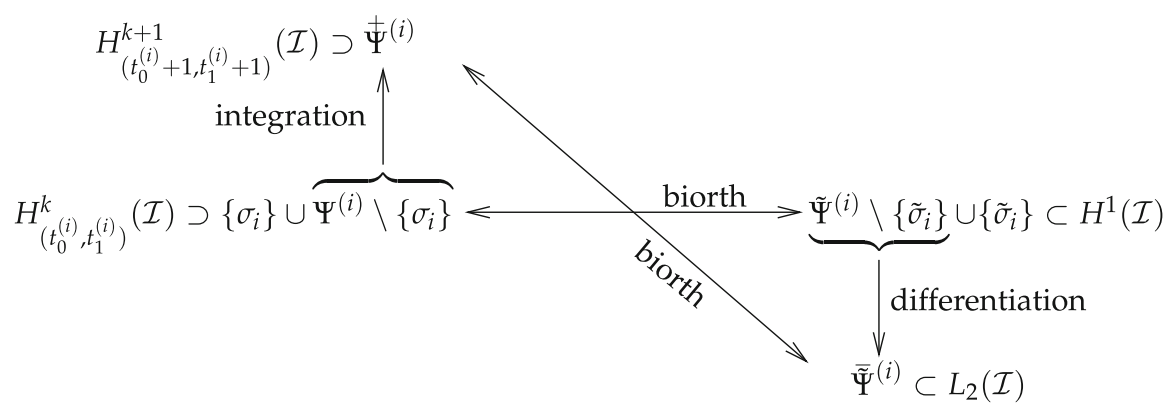

Fig. 1 Schematic relation between $\Psi^{(i)}, \tilde{\Psi}^{(i)}, \stackrel{+}{\Psi}^{(i)}$, and $\bar{\Psi}^{(i)}$

(3) $\left\{2^{-|\lambda|} \tilde{\psi}_{\lambda}^{(i)}: \lambda \in \nabla^{(i)}\right\}$ is a Riesz basis for $H^{1}(\mathcal{I})$,

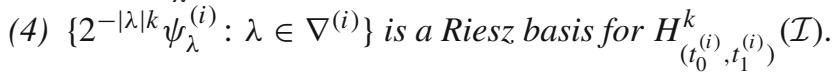

Here, as usual, $|\lambda| \in \mathbb{N}$ denotes the level of $\lambda \in \nabla^{(i)}$ (or that of $\psi_{\lambda}^{(i)}$ or $\tilde{\psi}_{\lambda}^{(i)}$ ).

Then setting, for $\lambda \in \stackrel{\circ}{ }^{(i)}:=\nabla^{(i)} \backslash\left\{\hat{\lambda}^{(i)}\right\}$,

$$
\bar{\psi}_{\lambda}^{(i)}:=x \mapsto \int_{0}^{x} 2^{|\lambda|} \psi_{\lambda}^{(i)}(y) d y, \quad \tilde{\psi}_{\lambda}^{(i)}:=-2^{-|\lambda|} \tilde{\psi}_{\lambda}^{(i)}{ }^{\prime},
$$

cf. Fig. 1, it holds that:

(5) $\overleftarrow{\Psi}^{(i)}=\left\{\hat{\psi}_{\lambda}^{(i)}: \lambda \in \stackrel{\circ}{\nabla}^{(i)}\right\}, \widetilde{\widetilde{\Psi}}^{(i)}=\left\{\widetilde{\psi}_{\lambda}^{(i)}: \lambda \in \stackrel{\circ}{\nabla}^{(i)}\right\}$ are $L_{2}(\mathcal{I})$-biorthogonal Riesz bases,

(6) $\left\{2^{-|\lambda|(k+1)} \stackrel{+}{\psi}_{\lambda}^{(i)}: \lambda \in \stackrel{\circ}{\nabla}^{(i)}\right\}$ is a Riesz basis for $H_{\left(t_{0}^{(i)}+1, t_{1}^{(i)}+1\right)}^{k+1}(\mathcal{I})$.

Moreover, supp $\tilde{\psi}_{\lambda}^{(i)} \subset \operatorname{supp} \tilde{\psi}_{\lambda}^{(i)}$, and supp $\bar{\psi}_{\lambda}^{(i)} \subset \operatorname{convhull}\left(\operatorname{supp} \psi_{\lambda}^{(i)}\right)$.

Conversely, when $\left(\Psi^{(i)}, \widetilde{\Psi}^{(i)}\right)$ satisfy (5)-(6), then setting $\nabla^{(i)}:=\stackrel{\circ}{\nabla}^{(i)} \cup\left\{\hat{\lambda}^{(i)}\right\}$, selecting a $\psi_{\hat{\lambda}^{(i)}}^{(i)}=\sigma_{i} \in H_{\left(t_{0}^{(i)}, t_{1}^{(i)}\right)}^{k}(\mathcal{I})$ with $\int_{\mathcal{I}} \sigma_{i} \neq 0$, taking $\tilde{\psi}_{\hat{\lambda}^{(i)}}^{(i)}=\tilde{\sigma}_{i}:=\mathbb{1} / \int_{\mathcal{I}} \sigma_{i}$, and, for $\lambda \in \stackrel{\circ}{\nabla}^{(i)}$, taking

$$
\begin{aligned}
& \psi_{\lambda}^{(i)}:=2^{-|\lambda|} \psi_{\lambda}^{(i)^{\prime}}, \quad \tilde{\psi}_{\lambda}^{(i)} \\
& :=x \mapsto-2^{|\lambda|}\left(\int_{0}^{x} \tilde{\psi}_{\lambda}^{(i)}(y) d y-\frac{\int_{0}^{1} \int_{0}^{z} \tilde{\psi}_{\lambda}^{(i)}(y) d y \sigma_{i}(z) d z}{\int_{0}^{1} \sigma_{i}(z) d z}\right),
\end{aligned}
$$

the conditions (1)-(4) are valid.

The relations indicated by the boxes are the analogues on the interval of (1.1) on the line.

Proof Either by (1), (2), and (4), or by the assumptions in the last paragraph of the theorem, we have $\left\langle\sigma_{i}, \tilde{\sigma}_{i}\right\rangle_{L_{2}(\mathcal{I})}=1, \sigma_{i} \in H_{\left(t_{0}^{(i)}, t_{1}^{(i)}\right)}^{k}(\mathcal{I})$, and $\tilde{\sigma}_{i} \in \operatorname{span}\{\mathbb{1}\}$. Consequently, 
$u \mapsto\left\langle u, \tilde{\sigma}_{i}\right\rangle_{L_{2}(\mathcal{I})} \sigma_{i}$ and $u \mapsto\left\langle u, \sigma_{i}\right\rangle_{L_{2}(\mathcal{I})} \tilde{\sigma}_{i}$ are projectors, which are bounded on $H_{\left(t_{0}^{(i)}, t_{1}^{(i)}\right)}^{k}(\mathcal{I})$ and $H^{1}(\mathcal{I})$, respectively. They give rise to the stable, biorthogonal decompositions $H_{\left(t_{0}^{(i)}, t_{1}^{(i)}\right)}^{k}(\mathcal{I})=\operatorname{span}\left\{\sigma_{i}\right\} \oplus\left(H_{\left(t_{0}^{(i)}, t_{1}^{(i)}\right)}^{k}(\mathcal{I}) \cap \operatorname{span}\{\mathbb{1}\}^{\perp L_{2}(\mathcal{I})}\right)$ and $H^{1}(\mathcal{I})=\operatorname{span}\{\mathbb{1}\} \oplus\left(H^{1}(\mathcal{I}) \cap \operatorname{span}\left\{\sigma_{i}\right\}^{\perp_{L_{2}}(\mathcal{I})}\right)$.

With this at hand, the conditions (1), (3), and (4) reduce to:

(i) $\left\{\psi_{\lambda}^{(i)}: \lambda \in \stackrel{\circ}{\nabla}^{(i)}\right\},\left\{\tilde{\psi}_{\lambda}^{(i)}: \lambda \in \stackrel{\circ}{\nabla}^{(i)}\right\}$ are $L_{2}(\mathcal{I})$-biorthogonal collections,

(ii) $\left\{2^{-|\lambda|} \tilde{\psi}_{\lambda}^{(i)}: \lambda \in \nabla^{(i)}\right\}$ is a Riesz basis for $H^{1}(\mathcal{I}) \cap \operatorname{span}\left\{\sigma_{i}\right\}^{\perp_{L_{2}}(\mathcal{I})}$,

(iii) $\left\{2^{-|\lambda| k} \psi_{\lambda}^{(i)}: \lambda \in \stackrel{\circ}{\nabla}^{(i)}\right\}$ is a Riesz basis for $H_{\left(t_{0}^{(i)}, t_{1}^{(i)}\right)}^{k}(\mathcal{I}) \cap \operatorname{span}\{\mathbb{1}\}^{\perp_{L_{2}(\mathcal{I})}}$.

The mapping $H^{1}(\mathcal{I}) \cap \operatorname{span}\left\{\sigma_{i}\right\}^{\perp_{L_{2}(\mathcal{I})}} \rightarrow L_{2}(\mathcal{I}): f \rightarrow f^{\prime}$ is bounded, with bounded inverse $g \mapsto\left(x \mapsto \int_{0}^{x} g(y) d y-\frac{\int_{0}^{1} \int_{0}^{z} g(y) \mathrm{d} y \sigma_{i}(z) d z}{\int_{0}^{1} \sigma_{i}(z) d z}\right)$. The mapping $H_{\left(t_{0}^{(i)}, t_{1}^{(i)}\right)}^{k}(\mathcal{I}) \cap \operatorname{span}\{\mathbb{1}\}^{\perp_{L_{2}(\mathcal{I})}} \rightarrow H_{\left(t_{0}^{(i)}+1, t_{1}^{(i)}+1\right)}^{k+1}(\mathcal{I}): g \mapsto\left(x \mapsto \int_{0}^{x} g(y) \mathrm{d} y\right)$ is bounded, with bounded inverse $f \mapsto f^{\prime}$. These facts show that the definitions (4.1) and (4.2) are equivalent. Furthermore, they show that (ii) is equivalent to $\tilde{\Psi}^{(i)}$ being a Riesz basis for $L_{2}(\mathcal{I})$, and that (iii) is equivalent to (6).

From (iii), or (6), we have $\stackrel{+}{\Psi}^{(i)} \subset H_{0}^{1}(\mathcal{I})$, and so for $\lambda, \mu \in \stackrel{\circ}{\nabla}^{(i)}$,

$$
\left\langle\bar{\psi}_{\lambda}^{(i)}, \tilde{\psi}_{\mu}^{(i)}\right\rangle_{L_{2}(\mathcal{I})}=\left\langle\bar{\psi}_{\lambda}^{(i)},-2^{-|\mu|} \tilde{\psi}_{\mu}^{(i)^{\prime}}\right\rangle_{L_{2}(\mathcal{I})}=2^{|\lambda|-|\mu|}\left\langle\psi_{\lambda}^{(i)}, \tilde{\psi}_{\mu}^{(i)}\right\rangle_{L_{2}(\mathcal{I})}
$$

i.e., (iii) is equivalent to biorthogonality of $\left(\overleftarrow{\Psi}^{(i)}, \overline{\tilde{\Psi}}^{(i)}\right)$.

Finally, both supp $\tilde{\psi}_{\lambda}^{(i)} \subset \operatorname{supp} \tilde{\psi}_{\lambda}^{(i)}$ and supp $\dot{\psi}_{\lambda}^{(i)} \subset$ convhull( $\operatorname{supp} \psi_{\lambda}^{(i)}$ ) follow from (4.1), for the latter using that $\int_{\mathcal{I}} \psi_{\lambda}^{(i)}=0$ by (iii).

Corollary 4.2 Assuming (1)-(4), then for $q \in\{1, \ldots, k+1\}$,

$$
\left\{2^{|\lambda|(1-q)} \psi_{\lambda}^{(i)}: \lambda \in \nabla^{(i)}\right\} \text { is a Riesz basis for } H_{\left(\min \left(t_{0}^{(i)}, q-1\right), \min \left(t_{1}^{(i)}, q-1\right)\right)}^{q-1}(\mathcal{I})
$$

and so $\left\{2^{|\lambda|(1-q)} \psi_{\lambda}^{(i)}: \lambda \in \stackrel{\circ}{\nabla}^{(i)}\right\}$ is a Riesz basis for this space intersected with $L_{2,0}(\mathcal{I})$; and, for $q \in\{0, \ldots, k+1\}$,

$$
\left\{2^{-|\lambda| q} \stackrel{+}{\psi}_{\lambda}^{(i)}: \lambda \in \stackrel{\circ}{\nabla}^{(i)}\right\} \text { is a Riesz basis for } H_{\left(\min \left(t_{0}^{(i)}+1, q\right), \min \left(t_{1}^{(i)}+1, q\right)\right)}^{q}(\mathcal{I})
$$

Proof Conditions (3) and (1) show that $\left\{2^{|\lambda|} \psi_{\lambda}^{(i)}: \lambda \in \nabla^{(i)}\right\}$ is a Riesz basis for $H^{1}(\mathcal{I})^{\prime}$. Together with (4), it shows that $\left\{2^{|\lambda|(1-q)} \psi_{\lambda}^{(i)}: \lambda \in \nabla^{(i)}\right\}$ is a Riesz basis for the interpolation space $\left[H^{1}(\mathcal{I})^{\prime}, H_{\left(t_{0}^{(i)}, t_{1}^{(i)}\right)}^{k}(\mathcal{I})\right]_{\frac{q}{k+1}, 2} \simeq H_{\left(\min \left(t_{0}^{(i)}, q-1\right), \min \left(t_{1}^{(i)}, q-1\right)\right)}^{q-1}(\mathcal{I})$ for $q \in\{1, \ldots, k+1\}$.

Properties (5)-(6) show that for $q \in\{0, \ldots, k+1\},\left\{2^{-|\lambda| q} \dot{\psi}_{\lambda}^{(i)}: \lambda \in \stackrel{\circ}{\nabla}^{(i)}\right\}$ is a Riesz basis for $\left[L_{2}(\mathcal{I}), H_{\left(t_{0}^{(i)}+1, t_{1}^{(i)}+1\right)}^{k+1}(\mathcal{I})\right]_{\frac{q}{k+1}, 2} \simeq H_{\left(\min \left(t_{0}^{(i)}+1, q\right), \min \left(t_{1}^{(i)}+1, q\right)\right)}^{q}(\mathcal{I})$. 
If $\Psi^{(i)}, \tilde{\Psi}^{(i)}$ that satisfy (1)-(4) are local, in the sense that diam supp $\psi_{\lambda}^{(i)} \lesssim 2^{-|\lambda|}$, diam supp $\tilde{\psi}_{\lambda}^{(i)} \lesssim 2^{-|\lambda|}$, then the same holds true for $\stackrel{+}{\Psi}^{(i)}$ and $\bar{\Psi}^{(i)}$ defined by (4.1). Alternatively, one may start with $\left(\Psi^{(i)}, \bar{\Psi}^{(i)}\right)$ that satisfy (5)-(6), and then, using a suitable $\sigma^{(i)}$, define $\Psi^{(i)}$ and $\tilde{\Psi}^{(i)}$ by (4.2). Following this approach, however, $\widetilde{\Psi}^{(i)}$ being local does not imply this property for $\tilde{\Psi}^{(i)}$.

Finally in this subsection, we note that it is well known how to construct pairs $\left(\Psi^{(i)}, \tilde{\Psi}^{(i)}\right)$ that satisfy (1)-(4) for any $k \in \mathbb{N}$ and $t_{0}^{(i)}, t_{1}^{(i)} \in\{0, \ldots, k\}$ and that additionally are local. We refer to the discussion in Sect. 5.1, where additionally the existence of suitable single-scale bases will be discussed.

\subsection{The (Anisotropic) Divergence-Free Wavelets}

For some $k \in \mathbb{N}$, and, for $1 \leq i \leq n$ and $b \in\{0,1\}, t_{b}^{(i)} \in\{0, \ldots, k\}$, from now on we fix biorthogonal pairs $\left(\Psi^{(i)}, \tilde{\Psi}^{(i)}\right)$ and $\left(\Psi^{(i)}, \widetilde{\tilde{\Psi}}^{(i)}\right)$ as in Theorem 4.1 .

Then the pairs $\left(\sigma_{i}, \tilde{\sigma}_{i}\right)$, and so the spaces $\mathbf{L}_{2,0}\left(\mathcal{I}^{S}\right)$ and $\tilde{\mathbf{L}}_{2,0}\left(\mathcal{I}^{S}\right)$ in Definition 3.1, and the embeddings $E^{(S)}, \tilde{E}^{(S)}$ and projectors $Q^{(S)}, \tilde{Q}^{(S)}$ in Definition 3.2 have all been determined. Upon setting $n_{b}^{(i)}:=\min \left(t_{b}^{(i)}+1, k\right)$, the spaces $\stackrel{\circ}{\mathbf{H}}^{k}\left(\mathcal{I}^{S}\right)$ in Definition 3.8 have been fixed as well.

Note that the conditions $\tilde{\sigma}_{i} \in \operatorname{span}\{\mathbb{1}\}$ and $\sigma_{i} \in H_{\left(t_{0}^{(i)}, t_{1}^{(i)}\right)}^{k}(\mathcal{I})$ required in Corollary 3.12 are guaranteed by Theorem 4.1 .

Using the pairs $\left(\Psi^{(i)} \backslash\left\{\sigma^{(i)}\right\}, \tilde{\Psi}^{(i)} \backslash\left\{\tilde{\sigma}^{(i)}\right\}\right)$ and $\left(\stackrel{\Psi}{\Psi}^{(i)}, \widetilde{\Psi}^{(i))}\right.$, in this subsection we construct, for any $S \subset\{1, \ldots, n\}$ with $\# S \geq 2$, bases $\boldsymbol{\Psi}_{\mathrm{df}}^{(S)}$ and $\tilde{\boldsymbol{\Psi}}_{\mathrm{df}}^{(S)}$ as needed in in Corollary 3.12. The key will be to make a Riesz basis $\boldsymbol{\Psi}^{(S)}=\left\{\boldsymbol{\psi}_{\lambda}^{(S)}: \lambda \in \nabla^{(S)}\right\}$ for $\mathbf{L}_{2,0}\left(\mathcal{I}^{S}\right) \cap \stackrel{\circ}{\mathbf{H}}^{k}\left(\mathcal{I}^{S}\right)$ with dual collection $\tilde{\boldsymbol{\Psi}}^{(S)} \subset \tilde{\mathbf{L}}_{2,0}\left(\mathcal{I}^{S}\right)$, such that $\nabla^{(S)}$ splits into two disjoint subsets, with the primals with indices from the first subset being divergence-fee and having vanishing normals at the boundary, and the duals with indices from the second subset being gradients.

For notational simplicity,

$$
\text { w.l.o.g., we consider } S=\{1, \ldots, n\} \text {. }
$$

Lemma 4.3 For $\lambda \in \nabla:=\stackrel{\circ}{\nabla}^{(1)} \times \cdots \times \stackrel{\circ}{\nabla}^{(n)}$ and $1 \leq i \leq n$, let

$$
\begin{aligned}
\underline{\psi}_{\lambda, i} & :=\psi_{\lambda_{1}}^{(1)} \otimes \cdots \otimes \psi_{\lambda_{i-1}}^{(i-1)} \otimes \dot{\psi}_{\lambda_{i}}^{(i)} \otimes \psi_{\lambda_{i+1}}^{(i+1)} \otimes \cdots \otimes \psi_{\lambda_{n}}^{(n)} \mathbf{e}_{i}, \\
\underline{\tilde{\psi}}_{\lambda, i}: & =\tilde{\psi}_{\lambda_{1}}^{(1)} \otimes \cdots \otimes \tilde{\psi}_{\lambda_{i-1}^{(i-1)}}^{(i)} \otimes \tilde{\psi}_{\lambda_{i}}^{(i)} \otimes \tilde{\psi}_{\lambda_{i+1}^{(i+1)}}^{(i)} \otimes \cdots \otimes \tilde{\psi}_{\lambda_{n}}^{(n)} \mathbf{e}_{i} .
\end{aligned}
$$

Then

$$
\left\{\left(\sum_{j=1}^{n} 4^{\left|\lambda_{j}\right| k}\right)^{-\frac{1}{2}} \underline{\psi}_{\lambda, i}: \lambda \in \nabla, 1 \leq i \leq n\right\},\left\{\left(\sum_{j=1}^{n} 4^{\left|\lambda_{j}\right| k}\right)^{\frac{1}{2}} \underline{\Psi}_{\lambda, i}: \lambda \in \nabla, 1 \leq i \leq n\right\},
$$


are $L_{2}\left(\mathcal{I}^{n}\right)^{n}$-biorthogonal collections; the first collection is a Riesz basis for $\mathbf{L}_{2,0}\left(\mathcal{I}^{n}\right) \cap \stackrel{\circ}{\mathbf{H}}^{k}\left(\mathcal{I}^{n}\right)$, and the second collection is in $\tilde{\mathbf{L}}_{2,0}\left(\mathcal{I}^{n}\right)$.

Proof The biorthogonality is obvious.

Let $1 \leq i \leq n$ be fixed. For $1 \leq j \leq n$, setting $p_{b}:=\left\{\begin{array}{ll}t_{b}^{(j)} & j \neq i \\ n_{b}^{(j)} & j=i\end{array}\right\}$,

$$
\left\{2^{-\left|\lambda_{j}\right| k} \psi_{\lambda_{1}}^{(1)} \otimes \cdots \otimes \psi_{\lambda_{i-1}}^{(i-1)} \otimes \stackrel{+}{\psi} \lambda_{\lambda_{i}}^{(i)} \otimes \psi_{\lambda_{i+1}}^{(i+1)} \otimes \cdots \otimes \psi_{\lambda_{n}}^{(n)}: \lambda \in \nabla\right\}
$$

is a Riesz basis for

$$
\{u \in L_{2}(\mathcal{I}) \otimes \cdots \otimes \underbrace{H_{\left(p_{0}, p_{1}\right)}^{k}(\mathcal{I})}_{j \text { th position }} \otimes \cdots \otimes L_{2}(\mathcal{I}): \int_{\mathcal{I}} u\left(x_{1}, \ldots, x_{n}\right) d x_{m}=0(m \neq i)\},
$$

by Corollary 4.2, and the definition of $n_{b}^{(j)}$ given at the beginning of this subsection. Consequently,

$$
\left\{\left(\sum_{j=1}^{n} 4^{\left|\lambda_{j}\right| k}\right)^{-\frac{1}{2}} \psi_{\lambda_{1}}^{(1)} \otimes \cdots \otimes \psi_{\lambda_{i-1}}^{(i-1)} \otimes \dot{\psi}_{\lambda_{i}}^{(i)} \otimes \psi_{\lambda_{i+1}}^{(i+1)} \otimes \cdots \otimes \psi_{\lambda_{n}}^{(n)}: \lambda \in \nabla\right\}
$$

is a Riesz basis for the intersection of these spaces over $1 \leq j \leq n$ (cf. [13, Proposition 2]), being

$$
\begin{aligned}
& \left\{u \in H^{k}\left(\mathcal{I}^{n}\right):\left.\partial_{x_{i}}^{p} u\right|_{x_{i}=b}=0\left(p \in\left\{0, \ldots, n_{b}^{(i)}-1\right\}\right) \quad(b \in\{0,1\}),\right. \\
& \left.\partial_{x_{j}}^{p} u\right|_{x_{j}=b}=0\left(p \in\left\{0, \ldots, t_{b}^{(j)}-1\right\}\right) \quad(b \in\{0,1\}, j \neq i), \\
& \left.\int_{\mathcal{I}} u\left(x_{1}, \ldots, x_{n}\right) d x_{j}=0 \quad(j \neq i)\right\} .
\end{aligned}
$$

In view of the definitions of $\mathbf{L}_{2,0}\left(\mathcal{I}^{n}\right), \tilde{\mathbf{L}}_{2,0}\left(\mathcal{I}^{n}\right)$, and $\stackrel{\circ}{\mathbf{H}}^{k}\left(\mathcal{I}^{n}\right)$, the proof is completed.

Next we are going to orthogonally transform the biorthogonal system of Lemma 4.3 into a new biorthogonal system that splits into two parts, with the primals from the first part being divergence-free, and the duals from the second part being gradients. This transformation generalizes upon the one that was used in the introduction to arrive at (1.5).

For any $\lambda \in \nabla$, we transform the biorthogonal system $\left\{\underline{\psi}_{\lambda, i}: 1 \leq i \leq n\right\}$, $\left\{\tilde{\tilde{\psi}}_{\lambda, i}: 1 \leq i \leq n\right\}$. We select an orthogonal $\mathbf{A}^{(\lambda)} \in \mathbb{R}^{n \times n}$ with its $n$th row given by 


$$
\mathbf{A}_{n \bullet}^{(\lambda)}=\boldsymbol{\alpha}^{(\lambda)^{\top}}, \quad \text { where } \boldsymbol{\alpha}^{(\lambda)}:=\left[2^{\left|\lambda_{1}\right|} \ldots 2^{\left|\lambda_{n}\right|}\right]^{\top} /\left(\sum_{i=1}^{n} 4^{\left|\lambda_{i}\right|}\right)^{\frac{1}{2}}
$$

An example of such a matrix $\mathbf{A}^{(\lambda)}$ is given by the Householder transformation

$$
\mathbf{A}^{(\lambda)}=I-\frac{2\left(\boldsymbol{\alpha}^{(\lambda)}-\boldsymbol{e}_{n}\right)\left(\boldsymbol{\alpha}^{(\lambda)}-\boldsymbol{e}_{n}\right)^{\top}}{\left(\boldsymbol{\alpha}^{(\lambda)}-\boldsymbol{e}_{n}\right)^{\top}\left(\boldsymbol{\alpha}^{(\lambda)}-\boldsymbol{e}_{n}\right)}
$$

which for $n=2,3$ reads as

$$
\left[\begin{array}{cc}
-\alpha_{2}^{(\lambda)} & \alpha_{1}^{(\lambda)} \\
\alpha_{1}^{(\lambda)} & \alpha_{2}^{(\lambda)}
\end{array}\right],\left[\begin{array}{ccc}
1-\frac{\left(\alpha_{1}^{(\lambda)}\right)^{2}}{1-\alpha^{(\lambda)}} & -\frac{\alpha_{1}^{(\lambda)} \alpha_{2}^{(\lambda)}}{1-\alpha_{3}^{(\lambda)}} & \alpha_{1}^{(\lambda)} \\
-\frac{\alpha_{1}^{(\lambda)} \alpha_{2}^{(\lambda)}}{1-\alpha_{3}^{(\lambda)}} & 1-\frac{\left(\alpha_{2}^{(\lambda)}\right)^{2}}{1-\alpha_{3}^{(\lambda)}} & \alpha_{2}^{(\lambda)} \\
\alpha_{1}^{(\lambda)} & \alpha_{2}^{(\lambda)} & \alpha_{3}^{(\lambda)}
\end{array}\right],
$$

respectively. The transformed system is now defined by

$$
\left[\begin{array}{c}
\psi_{\lambda, 1} \\
\vdots \\
\psi_{\lambda, n}
\end{array}\right]:=\mathbf{A}^{(\lambda)}\left[\begin{array}{c}
\underline{\psi}_{\lambda, 1} \\
\vdots \\
\underline{\psi}_{\lambda, n}
\end{array}\right], \quad\left[\begin{array}{c}
\tilde{\psi}_{\lambda, 1} \\
\vdots \\
\tilde{\psi}_{\lambda, n}
\end{array}\right]:=\mathbf{A}^{(\lambda)}\left[\begin{array}{c}
\tilde{\psi}_{\lambda, 1} \\
\vdots \\
\tilde{\psi}_{\lambda, n}
\end{array}\right] .
$$

Note that since $\mathbf{A}^{(\lambda)}$ applies to a group of basis functions that correspond to the same $\lambda$, this transformation does not affect possible locality of the basis functions.

Theorem 4.4 In the situation of Lemma 4.3, we have that

$$
\Psi_{\mathrm{df}}:=\left\{\left(\sum_{j=1}^{n} 4^{\left|\lambda_{j}\right| k}\right)^{-\frac{1}{2}} \boldsymbol{\psi}_{\lambda, i}: \lambda \in \nabla, 1 \leq i \leq n-1\right\}
$$

is a Riesz basis for $\mathbf{L}_{2,0}\left(\mathcal{I}^{n}\right) \cap \stackrel{\circ}{\mathbf{H}}^{k}\left(\mathcal{I}^{n}\right) \cap \mathbf{H}_{0}\left(\operatorname{div} 0 ; \mathcal{I}^{n}\right)$, with dual basis

$$
\tilde{\boldsymbol{\Psi}}_{\mathrm{df}}:=\left\{\left(\sum_{j=1}^{n} 4^{\left|\lambda_{j}\right| k}\right)^{\frac{1}{2}} \tilde{\boldsymbol{\psi}}_{\lambda, i}: \lambda \in \nabla, 1 \leq i \leq n-1\right\} \subset \tilde{\mathbf{L}}_{2,0}\left(\mathcal{I}^{n}\right) .
$$

Proof From $\mathbf{A}^{(\lambda)}$ being an orthogonal transformation, and the fact that the normalization factors of $\psi_{\lambda, i}$ and $\tilde{\psi}_{\lambda, i}$ in Lemma 4.3 are independent of $i$, this lemma shows that 


$$
\begin{aligned}
& \left\{\left(\sum_{j=1}^{n} 4^{\left|\lambda_{j}\right| k}\right)^{-\frac{1}{2}} \psi_{\lambda, i}: \lambda \in \nabla, 1 \leq i \leq n\right\}, \\
& \left\{\left(\sum_{j=1}^{n} 4^{\left|\lambda_{j}\right| k}\right)^{\frac{1}{2}} \tilde{\psi}_{\lambda, i}: \lambda \in \nabla, 1 \leq i \leq n\right\},
\end{aligned}
$$

are $L_{2}\left(\mathcal{I}^{n}\right)^{n}$-biorthogonal collections; the first collection is a Riesz basis for $\mathbf{L}_{2,0}\left(\mathcal{I}^{n}\right) \cap \stackrel{\circ}{\mathbf{H}}^{k}\left(\mathcal{I}^{n}\right)$, and the second collection is in $\tilde{\mathbf{L}}_{2,0}\left(\mathcal{I}^{n}\right)$.

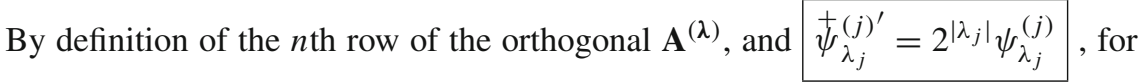
$1 \leq i \leq n-1$,

$$
\operatorname{div} \boldsymbol{\psi}_{\lambda, i}=\sum_{j=1}^{n} \mathbf{A}_{i j}^{(\lambda)} \operatorname{div} \underline{\psi}_{\lambda, j}=\left(\sum_{j=1}^{n} \mathbf{A}_{i j}^{(\lambda)} 2^{\left|\lambda_{j}\right|}\right) \psi_{\lambda_{1}}^{(1)} \otimes \cdots \otimes \psi_{\lambda_{n}}^{(n)}=0
$$

Since for $1 \leq i \leq n$, it holds that $\stackrel{+}{\Psi}^{(i)} \subset H_{0}^{1}(\mathcal{I})$, furthermore we have $\underline{\psi}_{\lambda, i} \cdot \mathbf{n}=0$ on $\partial \mathcal{I}^{n}$, and so $\psi_{\lambda, i} \cdot \mathbf{n}=0$ on $\partial \mathcal{I}^{n}$, which thus in particular holds true for $1 \leq i \leq n-1$.

From $2^{\left|\lambda_{j}\right|} \tilde{\psi}_{\lambda_{j}}^{(j)}=-\tilde{\psi}_{\lambda_{j}}^{(j)^{\prime}}$, it holds that

$$
\left(\sum_{j=1}^{n} 4^{\left|\lambda_{j}\right|}\right)^{\frac{1}{2}} \tilde{\psi}_{\lambda, n}=\sum_{j=1}^{n} 2^{\left|\lambda_{j}\right|} \tilde{\psi}_{\lambda, j}=-\operatorname{grad} \tilde{\psi}_{\lambda_{1}}^{(1)} \otimes \cdots \otimes \tilde{\psi}_{\lambda_{n}}^{(n)} .
$$

We infer that for $\mathbf{u} \in \mathbf{L}_{2,0}\left(\mathcal{I}^{n}\right) \cap \stackrel{\circ}{\mathbf{H}}^{k}\left(\mathcal{I}^{n}\right) \cap \mathbf{H}_{0}\left(\operatorname{div} 0 ; \mathcal{I}^{n}\right)$,

$$
\mathbf{u}=\sum_{\lambda \in \nabla} \sum_{i=1}^{n-1}\left\langle\mathbf{u},\left(\sum_{j=1}^{n} 4^{\left|\lambda_{j}\right| k}\right)^{\frac{1}{2}} \tilde{\boldsymbol{\psi}}_{\lambda, i}\right\rangle\left(\sum_{j=1}^{n} 4^{\left|\lambda_{j}\right| k}\right)^{-\frac{1}{2}} \boldsymbol{\psi}_{\lambda, i}
$$

and $\sum_{\lambda \in \nabla} \sum_{i=1}^{n-1}\left|\left\langle\mathbf{u},\left(\sum_{j=1}^{n} 4^{\left|\lambda_{j}\right| k}\right)^{\frac{1}{2}} \tilde{\boldsymbol{\psi}}_{\lambda, i}\right\rangle\right|^{2} \approx\|\mathbf{u}\|_{H^{k}\left(\mathcal{I}^{n}\right)^{n}}^{2}$, which completes the proof.

Together, Corollary 3.12 and Theorem 4.4 yield anisotropic wavelet Riesz bases for $\stackrel{\circ}{H}^{k}\left(\mathcal{I}^{n}\right) \cap \mathbf{H}_{0}\left(\operatorname{div} 0 ; \mathcal{I}^{n}\right)$ constructed from the biorthogonal pairs of univariate wavelet bases $\left(\Psi^{(i)}, \tilde{\Psi}^{(i)}\right)$ and $\left(\stackrel{\Psi}{\Psi}^{(i)}, \widetilde{\Psi}^{(i)}\right)$ from Theorem 4.1. We exemplify the construction for space dimensions $n=2$ and $n=3$.

For $n=2$, a similar construction was presented in $[15,16]$ based on the properties of the curl-operator (cf. Sect. 2). 
Example 4.5 For $n=2$,

$$
\left\{\frac{-2^{\left|\lambda_{2}\right|} \stackrel{+}{\psi}_{\lambda_{1}}^{(1)} \otimes \psi_{\lambda_{2}}^{(2)} \mathbf{e}_{1}+2^{\left|\lambda_{1}\right|} \psi_{\lambda_{1}}^{(1)} \otimes \stackrel{+}{\psi}_{\lambda_{2}}^{(2)} \mathbf{e}_{2}}{\left(4^{\left|\lambda_{1}\right| k}+4^{\left|\lambda_{2}\right| k}\right)^{\frac{1}{2}}\left(4^{\left|\lambda_{1}\right|}+4^{\left|\lambda_{2}\right|}\right)^{\frac{1}{2}}}:\left(\lambda_{1}, \lambda_{2}\right) \in \stackrel{\circ}{\nabla}^{(1)} \times \stackrel{\circ}{\nabla^{(2)}}\right\}
$$

is a Riesz basis for $\stackrel{\circ}{\mathbf{H}}^{k}\left(\mathcal{I}^{2}\right) \cap \mathbf{H}_{0}\left(\operatorname{div} 0 ; \mathcal{I}^{2}\right)$, with a dual basis given by

$$
\left\{\frac{-2^{\left|\lambda_{2}\right|} \tilde{\psi}_{\lambda_{1}}^{(1)} \otimes \tilde{\psi}_{\lambda_{2}}^{(2)} \mathbf{e}_{1}+2^{\left|\lambda_{1}\right|} \tilde{\psi}_{\lambda_{1}}^{(1)} \otimes \tilde{\psi}_{\lambda_{2}}^{(2)} \mathbf{e}_{2}}{\left(4^{\left|\lambda_{1}\right| k}+4^{\left|\lambda_{2}\right| k}\right)^{-\frac{1}{2}}\left(4^{\left|\lambda_{1}\right|}+4^{\left|\lambda_{2}\right|}\right)^{\frac{1}{2}}}:\left(\lambda_{1}, \lambda_{2}\right) \in \stackrel{\circ}{\nabla}^{(1)} \times \stackrel{\circ}{\nabla}^{(2)}\right\} \text {. }
$$

For $n=3$, with $\boldsymbol{\alpha}^{(\lambda)}$ as in (4.6) (reading $n=3$ ),

$$
\begin{aligned}
& \left\{\frac{\left(1-\frac{\left(\alpha_{1}^{(\lambda)}\right)^{2}}{1-\alpha_{3}^{(\lambda)}}\right) \dot{\psi}_{\lambda_{1}}^{(1)} \otimes \psi_{\lambda_{2}}^{(2)} \otimes \psi_{\lambda_{3}}^{(3)} \mathbf{e}_{1}-\frac{\alpha_{1}^{(\lambda)} \alpha_{2}^{(\lambda)}}{1-\alpha_{3}^{(\lambda)}} \psi_{\lambda_{1}}^{(1)} \otimes \dot{\psi}_{\lambda_{2}}^{(2)} \otimes \psi_{\lambda_{3}}^{(3)} \mathbf{e}_{2}+\alpha_{1}^{(\lambda)} \psi_{\lambda_{1}}^{(1)} \otimes \psi_{\lambda_{2}}^{(2)} \otimes \dot{\psi}_{\lambda_{3}}^{(3)} \mathbf{e}_{3}}{\left(4\left|\lambda_{1}\right| k+4\left|\lambda_{2}\right| k+4\left|\lambda_{3}\right| k\right)^{\frac{1}{2}}}\right. \\
& \left.:\left(\lambda_{1}, \lambda_{2}, \lambda_{3}\right) \in \stackrel{\circ}{\nabla}^{(1)} \times \stackrel{\circ}{\nabla}^{(2)} \times \stackrel{\circ}{\nabla}^{(3)}\right\} \\
& U \\
& \left\{\frac{\frac{\alpha_{1}^{(\lambda)} \alpha_{2}^{(\lambda)}}{\alpha_{3}^{(\lambda)}-1} \dot{\psi}_{\lambda_{1}}^{(1)} \otimes \psi_{\lambda_{2}}^{(2)} \otimes \psi_{\lambda_{3}}^{(3)} \mathbf{e}_{1}+\left(1-\frac{\left(\alpha_{2}^{(\lambda)}\right)^{2}}{1-\alpha_{3}^{(\lambda)}}\right) \psi_{\lambda_{1}}^{(1)} \otimes \dot{\psi}_{\lambda_{2}}^{(2)} \otimes \psi_{\lambda_{3}}^{(3)} \mathbf{e}_{2}+\alpha_{2}^{(\lambda)} \psi_{\lambda_{1}}^{(1)} \otimes \psi_{\lambda_{2}}^{(2)} \otimes \dot{\psi}_{\lambda_{3}}^{(3)} \mathbf{e}_{3}}{\left(4\left|\lambda_{1}\right| k+4\left|\lambda_{2}\right| k+4\left|\lambda_{3}\right| k\right)^{\frac{1}{2}}}\right. \\
& \left.:\left(\lambda_{1}, \lambda_{2}, \lambda_{3}\right) \in \stackrel{\circ}{\nabla}^{(1)} \times \stackrel{\circ}{\nabla}^{(2)} \times \stackrel{\circ}{\nabla}^{(3)}\right\} \\
& \bigcup\left\{\frac{-2^{\left|\lambda_{2}\right|} \stackrel{+}{\psi}_{\lambda_{1}}^{(1)} \otimes \psi_{\lambda_{2}}^{(2)} \otimes \sigma_{3} \mathbf{e}_{1}+2^{\left|\lambda_{1}\right|} \psi_{\lambda_{1}}^{(1)} \otimes \stackrel{+}{\psi}_{\lambda_{2}}^{(2)} \otimes \sigma_{3} \mathbf{e}_{2}}{\left(4^{\left|\lambda_{1}\right| k}+4^{\left|\lambda_{2}\right| k}\right)^{\frac{1}{2}}\left(4^{\left|\lambda_{1}\right|}+4^{\left|\lambda_{2}\right|}\right)^{\frac{1}{2}}}:\left(\lambda_{1}, \lambda_{2}\right) \in \stackrel{\circ}{\nabla}^{(1)} \times \stackrel{\circ}{\nabla}^{(2)}\right\} \\
& \bigcup\left\{\frac{-2^{\left|\lambda_{3}\right|} \stackrel{+}{\psi}_{\lambda_{1}}^{(1)} \otimes \sigma_{2} \otimes \psi_{\lambda_{3}}^{(3)} \mathbf{e}_{1}+2^{\left|\lambda_{1}\right|} \psi_{\lambda_{1}}^{(1)} \otimes \sigma_{2} \otimes \dot{+}_{\lambda_{3}}^{(3)} \mathbf{e}_{3}}{\left(4\left|\lambda_{1}\right| k+4\left|\lambda_{3}\right| k\right)^{\frac{1}{2}}\left(4\left|\lambda_{1}\right|+4\left|\lambda_{3}\right|\right)^{\frac{1}{2}}}:\left(\lambda_{1}, \lambda_{3}\right) \in \stackrel{\circ}{\nabla}^{(1)} \times \stackrel{\circ}{\nabla}^{(3)}\right\} \\
& \bigcup\left\{\frac{-2^{\left|\lambda_{3}\right|} \sigma_{1} \otimes \dot{\psi}_{\lambda_{2}}^{(2)} \otimes \psi_{\lambda_{3}}^{(3)} \mathbf{e}_{2}+2^{\left|\lambda_{2}\right|} \sigma_{1} \otimes \psi_{\lambda_{2}}^{(2)} \otimes \stackrel{+}{\psi}_{\lambda_{3}}^{(3)} \mathbf{e}_{3}}{\left(4^{\left|\lambda_{2}\right| k}+4^{\left|\lambda_{3}\right| k}\right)^{\frac{1}{2}}\left(4^{\left|\lambda_{2}\right|}+4^{\left|\lambda_{3}\right|}\right)^{\frac{1}{2}}}:\left(\lambda_{2}, \lambda_{3}\right) \in \stackrel{\circ}{\nabla}^{(2)} \times \stackrel{\circ}{\nabla}^{(3)}\right\}
\end{aligned}
$$

is a Riesz basis for $\stackrel{\circ}{\mathbf{H}}^{k}\left(\mathcal{I}^{3}\right) \cap \mathbf{H}_{0}\left(\operatorname{div} 0 ; \mathcal{I}^{3}\right)$. 
A dual basis is obtained by replacing, in all places, ${\stackrel{\psi}{\lambda_{i}}}_{\lambda_{i}}^{(i)}$ by $\tilde{\psi}_{\lambda_{i}}^{(i)}, \psi_{\lambda_{i}}^{(i)}$ by $\tilde{\psi}_{\lambda_{i}}^{(i)}$, and $\left(4^{\left|\lambda_{1}\right| k}+4^{\left|\lambda_{2}\right| k}+4^{\left|\lambda_{3}\right| k}\right)^{\frac{1}{2}},\left(4^{\left|\lambda_{1}\right| k}+4^{\left|\lambda_{2}\right| k}\right)^{\frac{1}{2}},\left(4^{\left|\lambda_{1}\right| k}+4^{\left|\lambda_{3}\right| k}\right)^{\frac{1}{2}}$, and $\left(4^{\left|\lambda_{2}\right| k}+4^{\left|\lambda_{3}\right| k}\right)^{\frac{1}{2}}$ by their reciprocals.

Remark 4.6 The biorthogonal collections constructed in Theorem 4.4 are clearly not unique. W.r.t. the splitting $\{1, \ldots, n\} \times \nabla=\{1, \ldots, n-1\} \times \nabla \bigcup\{n\} \times \nabla$, let us write the primal and dual bases from (4.8) as $\left[\begin{array}{l}\boldsymbol{\Psi}_{\mathrm{df}} \\ \boldsymbol{\Psi}_{\mathrm{gr}}\end{array}\right]$ and $\left[\begin{array}{c}\tilde{\boldsymbol{\Psi}}_{\mathrm{df}} \\ \tilde{\boldsymbol{\Psi}}_{\mathrm{gr}}\end{array}\right]$. Then for $A_{11}, A_{22}$ boundedly invertible, and $A_{21}$ bounded matrices (w.r.t. $\ell_{2}$ topologies and with the appropriate dimensions), the transformed systems $\left[\begin{array}{l}\underline{\boldsymbol{\Psi}}_{\mathrm{df}} \\ \underline{\boldsymbol{\Psi}}_{\mathrm{gr}}\end{array}\right]=\left[\begin{array}{cc}A_{11} & 0 \\ A_{21} & A_{22}\end{array}\right]\left[\begin{array}{l}\boldsymbol{\Psi}_{\mathrm{df}} \\ \boldsymbol{\Psi}_{\mathrm{gr}}\end{array}\right],\left[\begin{array}{l}\underline{\tilde{\Psi}}_{\mathrm{df}} \\ \underline{\boldsymbol{\Psi}}_{\mathrm{gr}}\end{array}\right]=$ $\left[\begin{array}{cc}A_{11}^{-\top}-A_{11}^{-\top} A_{12}^{\top} A_{22}^{-\top} \\ 0 & A_{22}^{-\top}\end{array}\right]\left[\begin{array}{c}\tilde{\boldsymbol{\Psi}}_{\mathrm{df}} \\ \tilde{\boldsymbol{\Psi}}_{\mathrm{gr}}\end{array}\right]$ share the properties with the original systems. That is, they are biorthogonal, $\left[\begin{array}{l}\underline{\boldsymbol{\Psi}}_{\mathrm{df}} \\ \underline{\boldsymbol{\Psi}}_{\mathrm{gr}}\end{array}\right]$ is a Riesz basis for $\mathbf{L}_{2,0}\left(\mathcal{I}^{n}\right) \cap \stackrel{\circ}{\mathbf{H}}^{k}\left(\mathcal{I}^{n}\right),\left[\begin{array}{l}\underline{\tilde{\boldsymbol{\Psi}}}_{\mathrm{df}} \\ \underline{\tilde{\boldsymbol{\Psi}}}_{\mathrm{gr}}\end{array}\right] \subset$ $\tilde{\mathbf{L}}_{2,0}\left(\mathcal{I}^{n}\right), \underline{\boldsymbol{\Psi}}_{\mathrm{df}} \subset \mathbf{H}_{0}\left(\operatorname{div} 0 ; \mathcal{I}^{n}\right)$, and $\underline{\tilde{\Psi}}_{\mathrm{gr}} \subset \operatorname{grad} H^{1}\left(\mathcal{I}^{n}\right)$. Consequently, $\underline{\boldsymbol{\Psi}}_{\mathrm{df}}$ is a Riesz basis for $\mathbf{L}_{2,0}\left(\mathcal{I}^{n}\right) \cap \stackrel{\circ}{\mathbf{H}}^{k}\left(\mathcal{I}^{n}\right) \cap \overline{\mathbf{H}}_{0}\left(\operatorname{div} 0 ; \mathcal{I}^{n}\right)$ with dual basis $\underline{\tilde{\Psi}}_{\mathrm{df}} \subset \tilde{\mathbf{L}}_{2,0}\left(\mathcal{I}^{n}\right)$.

\section{Single-Scale Bases, and Isotropic Divergence-Free Wavelets}

To compute an approximation to a function $\mathbf{u} \in \stackrel{\circ}{\mathbf{H}}^{k}\left(\mathcal{I}^{n}\right) \cap \mathbf{H}_{0}\left(\operatorname{div} 0 ; \mathcal{I}^{n}\right)$ from our divergence-free wavelet basis, one has to select a subset of its infinite index set. For sufficiently smooth $\mathbf{u}$, choosing a so-called sparse-grid index set, reading as the union of all indices $\left(\lambda_{1}, \ldots, \lambda_{n}\right)$ that for some $\ell \in \mathbb{N}$ satisfy $\sum_{i=1}^{m}\left|\lambda_{i}\right| \leq \ell$ (where the absolute values of "lacking coordinates" $\lambda_{i}$, cf. (4.12)-(4.14), should read as zero), the approximation error in $H^{k}\left(\mathcal{I}^{n}\right)^{n}$ is of order $N^{-(d+1-k)}$, up to $\log$-factors. Here $N$ is the cardinality of the sparse-grid index set, and $d$ is the order of the univariate wavelets. For $k>0$, the log-factors can even be avoided by applying a somewhat modified index set known as an optimized sparse grid ([12], [10, Ch.4]). These results should be compared with the generally best possible approximation error realized with isotropic approximation which is of order $N^{-\frac{d+1-k}{n}}$.

When $\mathbf{u}$ is the solution of a PDE, say the (Navier-) Stokes equations, then a near-best approximation from the span of the wavelets with indices in a given index subset is given by its corresponding Galerkin approximation. Since the basis is Riesz, the stiffness matrix is well-conditioned, uniformly in the index subset. Consequently, the Galerkin system can be solved by the application of a simple iterative scheme. Due to the anisotropic nature of the basis functions, the stiffness matrix is, however, generally far from being sparse. Nevertheless, for (optimized) sparse grid index sets, the application of this matrix to a vector can be computed in linear complexity using the so-called unidirectional approach [1]. This approach relies on the availability of locally supported single scale bases and corresponding refinement relations for the univariate wavelets that are used as building blocks of the divergence-free wavelets. This is the topic of Sect. 5.1. 
The above considerations apply under the assumption that $\mathbf{u}$ is sufficiently smooth. For a much larger class of functions, an error of order $N^{-(d+1-k)}$ can be realized by a Galerkin approximation w.r.t. an adaptively created index set [6]. For index sets that have a certain multi-tree structure, the application of the stiffness matrix to a vector can still be applied in linear complexity [17].

Although the application of anisotropic basis functions clearly offers important advantages, it is fair to say that their implementation is more demanding than that of isotropic basis functions. Therefore, in Sect. 5.2, a construction will be presented of an isotropic Riesz basis for $\stackrel{\circ}{H}^{k}\left(\mathcal{I}^{2}\right) \cap \mathbf{H}_{0}\left(\operatorname{div} 0 ; \mathcal{I}^{2}\right)$, so unfortunately only for $n=$ 2.

\subsection{Single Scale Bases and Refinement Equations}

In this subsection, we fix the index $1 \leq i \leq n$, and drop it from our notations.

Assuming that biorthogonal (locally supported) single scale bases are available for the univariate primal and dual wavelet collections $\Psi$ and $\tilde{\Psi}$, in this section we construct biorthogonal (locally supported) single scale bases for $\vec{\Psi}$ and $\widetilde{\Psi}$.

Proposition 5.1 (single-scale bases) Let $\Psi, \tilde{\Psi}, \stackrel{+}{\Psi}, \widetilde{\Psi}$ be as in Theorem 4.1, and so in particular with $\tilde{\sigma}=\tilde{\psi}_{\hat{\lambda}} \in \operatorname{span}\{\mathbb{1}\}$. W.l.o.g. we may assume that $|\hat{\lambda}|=0$.

(a). For $\ell \in \mathbb{N}$, let

$$
\begin{aligned}
& \Phi_{\ell}=\left\{\phi_{\lambda}: \lambda=(|\lambda|, q) \in \Delta_{\ell}:=\{\ell\} \times\left\{1, \ldots N_{\ell}\right\}\right\}, \\
& \tilde{\Phi}_{\ell}=\left\{\tilde{\phi}_{\lambda}: \lambda \in \Delta_{\ell}\right\}
\end{aligned}
$$

be biorthogonal bases for $\left\{\psi_{\lambda}: \lambda \in \nabla,|\lambda| \leq \ell\right\}, \operatorname{span}\left\{\tilde{\psi}_{\lambda}: \lambda \in \nabla,|\lambda| \leq \ell\right\}$, scaled such that for each $\ell$,

$$
\int_{\mathcal{I}} \phi_{\lambda} \quad \text { is independent of } \lambda \in \Delta_{\ell}
$$

Then

$$
\begin{aligned}
& \overleftarrow{\Phi}_{\ell}=\left\{\stackrel{+}{\phi}_{\lambda}: \lambda=(\ell, q) \in \stackrel{\circ}{\Delta}_{\ell}:=\{\ell\} \times\left\{1, \ldots N_{\ell}-1\right\}\right\}, \\
& \widetilde{\Phi}_{\ell}=\left\{\widetilde{\phi}_{\lambda}: \lambda \in \stackrel{\circ}{\Delta}_{\ell}\right\}
\end{aligned}
$$

defined by

$$
\stackrel{+}{\phi}_{\ell, q}:=x \mapsto \int_{0}^{x} 2^{\ell+1}\left(\phi_{\ell, q+1}(y)-\phi_{\ell, q}(y)\right) d y, \quad \tilde{\phi}_{\ell, q}:=-2^{-(\ell+1)} \sum_{p=q+1}^{N_{\ell}} \tilde{\phi}_{\ell, p}^{\prime},
$$

are biorthogonal bases for $\operatorname{span}\left\{\stackrel{+}{\psi}_{\lambda}: \lambda \in \stackrel{\circ}{\nabla},|\lambda| \leq \ell\right\}, \operatorname{span}\left\{\tilde{\psi}_{\lambda}: \lambda \in \stackrel{\circ}{\nabla},|\lambda| \leq \ell\right\}$. 
(b). In addition, let $\left\|\phi_{\ell, q}\right\|_{L_{2}(\mathcal{I})},\left\|\tilde{\phi}_{\ell, q}\right\|_{L_{2}(\mathcal{I})} \lesssim 1$, and let $\Phi_{\ell}, \tilde{\Phi}_{\ell}$ be uniformly local and locally finite, meaning that the diameters of the supports of the basis functions are of order $2^{-\ell}$, and that each interval of length $2^{-\ell}$ intersects the supports of a (uniformly) bounded number of basis functions.

Then, numbering the basis functions such that inf $\operatorname{supp} \phi_{\ell, q} \leq \inf \operatorname{supp} \phi_{\ell, q+1}$, it holds that $\Phi_{\ell}, \bar{\Phi}_{\ell}$ are uniformly local and locally finite. Moreover, $\Phi_{\ell}, \tilde{\Phi}_{\ell}, \bar{\Phi}_{\ell}, \bar{\Phi}_{\ell}$ are uniform $L_{2}(\mathcal{I})$-Riesz bases for their spans. E.g., for $\Phi_{\ell}$, the latter means that $\left\|\mathbf{c}^{\top} \Phi_{\ell}\right\|_{L_{2}(\mathcal{I})} \approx\|\mathbf{c}\|\left(\mathbf{c} \in \mathbb{R}^{N_{\ell}-1}\right)$.

Proof (a). An application of a basis transform shows that

$$
\left\{\phi_{\ell, 1}, \phi_{\ell, 2}-\phi_{\ell, 1}, \ldots, \phi_{\ell, N_{\ell}}-\phi_{\ell, N_{\ell}-1}\right\}, \quad\left\{\sum_{q=1}^{N_{\ell}} \tilde{\phi}_{\ell, q}, \sum_{q=2}^{N_{\ell}} \tilde{\phi}_{\ell, q}, \ldots, \tilde{\phi}_{\ell, N_{\ell}}\right\}
$$

are biorthogonal bases for span $\Phi_{\ell}$, span $\tilde{\Phi}_{\ell}$.

Because of $\int_{\mathcal{I}} \phi_{\ell, q+1}-\phi_{\ell, q}=0$ by $(5.1)$, and so $\Phi_{\ell} \subset H_{0}^{1}(\mathcal{I})$, integration by parts now shows that $\Phi_{\ell}, \tilde{\Phi}_{\ell}$ are biorthogonal.

Again (5.1) and $\mathbb{1} \in \operatorname{span} \tilde{\Phi}_{\ell}$ show that $\sum_{q=1}^{N_{\ell}} \tilde{\phi}_{\ell, q} \in \operatorname{span}\{\mathbb{1}\}$. So for $\lambda \in \stackrel{\circ}{\nabla}$, $|\lambda| \leq \ell$, we have $\psi_{\lambda} \in \operatorname{span}\left\{\phi_{\ell, 2}-\phi_{\ell, 1}, \ldots, \phi_{\ell, N_{\ell}}-\phi_{\ell, N_{\ell}-1}\right\}$, and so $\psi_{\lambda} \in \operatorname{span} \Phi_{\ell}$.

Finally, since $\sum_{q=1}^{N_{\ell}} \tilde{\phi}_{\ell, q}^{\prime}=0$, for $\lambda \in \stackrel{\circ}{\nabla},|\lambda| \leq \ell$ we have $\tilde{\psi}_{\lambda} \in \operatorname{span} \tilde{\Phi}_{\ell}$.

(b). By supp $\phi_{\ell, q} \subset$ convhull( $\operatorname{supp} \phi_{\ell, q+1} \cup \operatorname{supp} \phi_{\ell, q}$ ) by (5.1), the assumption on the numbering of the basis functions, and $\Phi_{\ell}$ being uniformly local and locally finite, we have that $\Phi_{\ell}$ is uniformly local and locally finite. Together with $\left\|\phi_{\ell, q}\right\|_{L_{2}(\mathcal{I})} \lesssim 1$, it shows that $\left\|\dot{\phi}_{\ell, q}\right\|_{L_{2}(\mathcal{I})} \lesssim 1$, and so

$$
\left\|\mathbf{c}^{\top} \Phi_{\ell}\right\|_{L_{2}(\mathcal{I})} \lesssim\|\mathbf{c}\|
$$

By the assumption on the numbering of the basis functions, supp $\tilde{\phi}_{\ell, q} \cap \operatorname{supp} \phi_{\ell, q} \neq$ $\emptyset$, and the uniform locality and local finiteness of $\Phi_{\ell}$ and $\tilde{\Phi}_{\ell}$, it follows that at the left of some neighborhood with diameter of order $2^{-\ell}$ of $\operatorname{supp} \tilde{\phi}_{\ell, q}$, the function $\sum_{p=q+1}^{N_{\ell}} \tilde{\phi}_{\ell, p}$ is identically zero, whereas at the right of this neighborhood it is equal to $\sum_{p=1}^{N_{\ell}} \tilde{\phi}_{\ell, p}$. From $\sum_{p=1}^{N_{\ell}} \tilde{\phi}_{\ell, p}^{\prime}=0$, it follows that $\widetilde{\Phi}_{\ell}$ is uniformly local and locally finite.

Any $u \in \operatorname{span} \tilde{\Phi}_{\ell}$ can be written as $\sum_{\{\lambda \in \nabla:|\lambda| \leq \ell\}} c_{\lambda} 2^{-|\lambda|} \tilde{\psi}_{\lambda}$. From $\left\{2^{-|\lambda|} \tilde{\psi}_{\lambda}: \lambda \in\right.$ $\nabla\}$ and $\tilde{\Psi}$ being Riesz bases for $H^{1}(\mathcal{I})$ and $L_{2}(\mathcal{I})$, respectively, by Corollary 4.2, we have $\|u\|_{H^{1}(\mathcal{I})}^{2} \approx \sum_{\{\lambda \in \nabla:|\lambda| \leq \ell\}}\left|c_{\lambda}\right|^{2} \leq 4^{\ell} \sum_{\{\lambda \in \nabla:|\lambda| \leq \ell\}}\left|c_{\lambda} 2^{-|\lambda|}\right|^{2} \lesssim 4^{\ell}\|u\|_{L_{2}(\mathcal{I})}^{2}$. So on span $\tilde{\Phi}_{\ell}$, a so-called inverse inequality applies.

Writing $\overline{\tilde{\phi}}_{\ell, q}:=-\left.2^{-(\ell+1)} \sum_{p=q+1}^{N_{\ell}} \tilde{\phi}_{\ell, p}^{\prime}\right|_{\text {supp }} \overline{\tilde{\phi}}_{\ell, q}$, and noting that the number of nonzero terms is uniformly bounded, we conclude that $\left\|\widetilde{\phi}_{\ell, q}\right\|_{L_{2}(\mathcal{I})} \lesssim 1$. From $\bar{\Phi}_{\ell}$ being uniformly local and locally finite, we infer

$$
\left\|\mathbf{c}^{\top} \widetilde{\Phi}_{\ell}\right\|_{L_{2}(\mathcal{I})} \lesssim\|\mathbf{c}\|
$$


Given a vector $\mathbf{c}=\left(c_{q}\right)_{q}$, let $u=\mathbf{c}^{\top} \stackrel{\Phi}{\ell}_{\ell}$. Then $\left.\left|c_{q}\right|=\left|\left\langle u, \tilde{\bar{\phi}}_{\ell, q}\right\rangle_{L_{2}(\mathcal{I})}\right| \lesssim\|u\|_{L_{2}(\operatorname{supp}} \tilde{\phi}_{\ell, q}\right)$. From $\widetilde{\Phi}_{\ell}$ being uniformly locally finite, we conclude that $\|\mathbf{c}\| \lesssim\left\|\mathbf{c}^{\top} \Phi_{\ell}\right\|_{L_{2}(\mathcal{I})}$. Similarly, (5.3) shows that $\|\mathbf{c}\| \lesssim\left\|\mathbf{c}^{\top} \bar{\Phi}_{\ell}\right\|_{L_{2}(\mathcal{I})}$.

The proof of $\Phi_{\ell}, \tilde{\Phi}_{\ell}$ being uniform $L_{2}(\mathcal{I})$-Riesz bases for their spans is similar.

Proposition 5.2 (refinement equations) In the situation of Proposition 5.1(a), let $\mathbf{M}_{\ell}=\left[\begin{array}{ll}\mathbf{M}_{\ell, 0} & \mathbf{M}_{\ell, 1}\end{array}\right]$ with $\mathbf{M}_{\ell, 0} \in \mathbb{R}^{N_{\ell+1} \times N_{\ell}}, \mathbf{M}_{\ell, 1} \in \mathbb{R}^{N_{\ell+1} \times\left(N_{\ell+1}-N_{\ell}\right)}$ be such that

$$
\left[\begin{array}{ll}
\Phi_{\ell}^{\top} & \Psi_{\ell+1}^{\top}
\end{array}\right]=\Phi_{\ell+1}^{\top} \mathbf{M}_{\ell}
$$

where $\Psi_{\ell+1_{+}}:=\left\{\psi_{\lambda}: \lambda \in \nabla,|\lambda|=\ell+1\right\}$.

Putting $\Psi_{\ell+1}:=\left\{\dot{\psi}_{\lambda}: \lambda \in \stackrel{\nabla}{ },|\lambda|=\ell+1\right\}$, and defining $\mathbf{A}_{\ell} \in \mathbb{R}^{N_{\ell} \times\left(N_{\ell}-1\right)}$ by

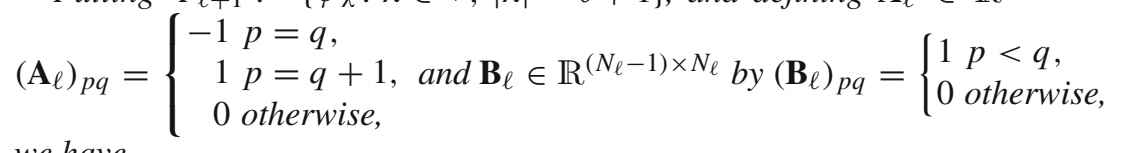
we have

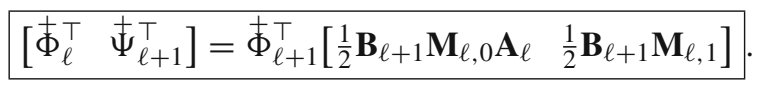

Proof We set $\underline{\Phi}_{\ell}=\left[x \mapsto \int_{0}^{x} 2^{\ell+1} \phi_{\ell, 1}(y) \mathrm{d} y \quad \stackrel{\Phi}{\ell}_{\ell}^{\top}\right]^{\top}$, and $\mathbf{T}_{\ell}=\left[\begin{array}{ccc}1 & & \\ -1 & 1 & \\ & \ddots & \\ & -1 & 1\end{array}\right] \in$ $\mathbb{R}^{N_{\ell} \times N_{\ell}}$. From $\underline{\Phi}_{\ell}(x)=2^{\ell+1} \mathbf{T}_{\ell} \int_{0}^{x} \Phi_{\ell}(z) d z$ and $\overleftarrow{\Psi}_{\ell}(x)=2^{\ell} \int_{0}^{x} \Psi_{\ell}(z) d z$, we infer that

$$
\left[\begin{array}{ll}
\underline{\Phi}_{\ell}^{\top} & \overleftarrow{\Psi}_{\ell+1}^{\top}
\end{array}\right]=\underline{\Phi}_{\ell+1}^{\top}\left[\frac{1}{2} \mathbf{T}_{\ell+1}^{-\top} \mathbf{M}_{\ell, 0} \mathbf{T}_{\ell}^{\top} \quad \frac{1}{2} \mathbf{T}_{\ell+1}^{-\top} \mathbf{M}_{\ell, 1}\right]
$$

By deleting the first column of $\frac{1}{2} \mathbf{T}_{\ell+1}^{-\top} \mathbf{M}_{\ell, 0} \mathbf{T}_{\ell}^{\top}$, and by realizing that by span $\Phi_{\ell} \cup \bar{\Psi}_{\ell+1}$ $=\operatorname{span} \stackrel{\Phi}{\ell+1}_{\ell}$, and $\underline{\Phi}_{\ell+1}$ being independent, the first row of $\frac{1}{2} \mathbf{T}_{\ell+1}^{-\top} \mathbf{M}_{\ell, 0} \mathbf{T}_{\ell}^{\top}$, without its first column, and that of $\frac{1}{2} \mathbf{T}_{\ell+1}^{-\top} \mathbf{M}_{\ell, 1}$ are zero, we get the expressions of $\stackrel{\Phi}{\ell}_{\ell}^{\top}$ and $\Psi_{\ell+1}^{\top}$ in terms of $\Phi_{\ell+1}^{\top}$.

Remark 5.3 Biorthogonality shows that in the situation of Proposition 5.2, we also have

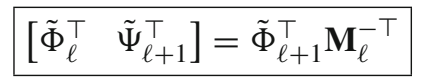

and

$$
\left[\begin{array}{ll}
\overline{\tilde{\Phi}}_{\ell}^{\top} & \overline{\tilde{\Psi}}_{\ell+1}^{\top}
\end{array}\right]=\overline{\tilde{\Phi}}_{\ell+1}^{\top}\left[\frac{1}{2} \mathbf{B}_{\ell+1} \mathbf{M}_{\ell, 0} \mathbf{A}_{\ell} \quad \frac{1}{2} \mathbf{B}_{\ell+1} \mathbf{M}_{\ell, 1}\right]^{-\top}
$$

In the situation of Proposition 5.1(b), all these four basis transformations are uniformly "sparse" (despite of the fact that $\mathbf{B}_{\ell}$ is not sparse), and uniformly well-conditioned. 
At this point, we note that for any $k \in \mathbb{N}_{0}$ and $t_{0}, t_{1} \in\{0, \ldots, k\}, \Psi, \tilde{\Psi}$ as in Theorem 4.1, with corresponding single-scale bases $\Phi_{\ell}, \tilde{\Phi}_{\ell}$ as in Proposition 5.1 have been constructed in [5]. Choosing $\mathbb{N} \ni \tilde{d} \geq d>k$, with $d+\tilde{d}$ even, and $\tilde{d}$ sufficiently large to ensure Theorem 4.1(3) (valid choices are, e.g., $(d, \tilde{d})=(2,4),(3,7),(4,10))$, span $\Phi_{\ell}$ is the space of splines of order $d$ w.r.t. knot sequence

$$
(\underbrace{0, \ldots, 0}_{d-t_{0} \text { times }}, r 2^{-\ell},(r+1) 2^{-\ell}, \ldots, 1-r 2^{-\ell}, \underbrace{1, \ldots, 1}_{d-t_{1} \text { times }}),
$$

for some $\mathbb{N} \ni r \geq d-1$.

The dual space $\operatorname{span} \tilde{\Phi}_{\ell}$ is a subspace of $\operatorname{span}\left\{\left._{d, \tilde{d}} \tilde{\phi}\left(2^{\ell} \cdot-m\right)\right|_{[0,1]}: m \in \mathbb{Z}\right\}$, where, with ${ }_{d} \phi$ denoting the cardinal B-spline of order $d,{ }_{d, \tilde{d}} \tilde{\phi}$ is a compactly supported refinable function with $\left.{ }_{d} \phi,{ }_{d, \tilde{d}} \tilde{\phi}(\cdot-m)\right\rangle_{L_{2}(\mathbb{R})}=\delta_{0, m}(m \in \mathbb{Z})$ constructed in [4]. Since it holds that $P_{\tilde{d}-1} \subset$ span $\tilde{\Phi}_{0}$, in particular we have that $\mathbb{1} \in \operatorname{span} \tilde{\Phi}_{0}$. After making a simple basis transformation, which involves only primal and dual wavelets at the coarsest level, we have that for some $\hat{\lambda} \in \nabla$ with $|\hat{\lambda}|=0, \tilde{\psi}_{\hat{\lambda}} \in \operatorname{span} \mathbb{1}$, as required.

In [3,23], [10, Ch. 2], modified constructions were proposed yielding quantitatively better conditioned bases. With these constructions, span $\Phi_{\ell}$ is the space of splines of order $d$ w.r.t. uniform knot sequence

$$
(\underbrace{0, \ldots, 0}_{d-t_{0} \text { times }}, 2^{-\ell}, \ldots, 1-2^{-\ell}, \underbrace{1, \ldots, 1}_{d-t_{1} \text { times }}) .
$$

Refinement matrices $\mathbf{M}_{\ell, 0}$ and $\mathbf{M}_{\ell, 1}$, as defined in Proposition 5.2, which determine the whole construction of the anisotropic (and forthcoming isotropic) divergence-free wavelet basis, can be found in these references.

Finally in this subsection, recall that the collection $\Phi_{\ell}\left(\tilde{\Phi}_{\ell}\right)$ is not a basis for $\left\{\psi_{\lambda}: \lambda \in \stackrel{\nabla}{\nabla},|\lambda| \leq \ell\right\}\left(\left\{\tilde{\psi}_{\lambda}: \lambda \in \stackrel{\nabla}{\nabla},|\lambda| \leq \ell\right\}\right)$, but for the slightly bigger space $\left\{\psi_{\lambda}: \lambda \in \nabla,|\lambda| \leq \ell\right\}\left(\left\{\tilde{\psi}_{\lambda}: \lambda \in \nabla,|\lambda| \leq \ell\right\}\right)$. This is not a problem for the application of multi-to-single scale transforms to facilitate an efficient application of a stiffness matrix in (anisotropic) wavelets coordinates. In order to construct isotropic divergence-free wavelet bases, however, biorthogonal single scale bases for the smaller spaces will be needed, which issue we take up now.

From (5.2), and $\sum_{q=1}^{N_{\ell}} \tilde{\phi}_{\ell, q} \in \operatorname{span}\{\mathbb{1}\}$, it follows that

$$
\Theta_{\ell}=\left\{\theta_{\ell, q}:=\phi_{\ell, q+1}-\phi_{\ell, q}:(\ell, q)=\lambda \in \stackrel{\circ}{\Delta}_{\ell}\right\}
$$

is a basis for $\operatorname{span}\left\{\psi_{\lambda}: \lambda \in \stackrel{\circ}{\nabla},|\lambda| \leq \ell\right\}=\operatorname{span}\left\{\psi_{\lambda}: \lambda \in \nabla,|\lambda| \leq \ell\right\} \cap \mathbb{1}^{\perp}$. Let

$$
\tilde{\Theta}_{\ell}=\left\{\tilde{\theta}_{\lambda}: \lambda \in \stackrel{\circ}{\Delta}_{\ell}\right\}
$$

denote the basis for $\operatorname{span}\left\{\tilde{\psi}_{\lambda}: \lambda \in \stackrel{\circ}{\nabla},|\lambda| \leq \ell\right\}$ that is biorthogonal to $\Theta_{\ell}$. 
It holds that

$$
\stackrel{+}{\phi}_{\lambda}^{\prime}=2^{|\lambda|+1} \theta_{\lambda}, \quad \tilde{\theta}_{\lambda}^{\prime}=-2^{|\lambda|+1} \widetilde{\phi}_{\lambda} \quad\left(\lambda \in \stackrel{\circ}{\Delta}_{\ell}\right)
$$

where the first equality follows from the definitions of $\stackrel{+}{\Phi}_{\ell}$ and $\Theta_{\ell}$. The second equation is a consequence of

$\delta_{\lambda \mu}=\left\langle\theta_{\lambda}, \tilde{\theta}_{\mu}\right\rangle_{L_{2}(\mathcal{I})}=2^{-(|\lambda|+1)}\left\langle\dot{\phi}_{\lambda}{ }^{\prime}, \tilde{\theta}_{\mu}\right\rangle_{L_{2}(\mathcal{I})}=-2^{-(|\lambda|+1)}\left\langle\dot{\phi}_{\lambda}, \tilde{\theta}_{\mu}^{\prime}\right\rangle_{L_{2}(\mathcal{I})} \quad\left(\lambda, \mu \in \stackrel{\circ}{\Delta}_{\ell}\right)$,

the fact that $\tilde{\theta}_{\mu}^{\prime} \underset{\tilde{\tilde{\Phi}}}{\stackrel{(4.1)}{\in}} \operatorname{span}\left\{\tilde{\psi}_{\lambda}: \lambda \in \stackrel{\circ}{\nabla},|\lambda| \leq \ell\right\}=\operatorname{span}\left\{\tilde{\phi}_{\lambda}: \lambda \in \stackrel{\circ}{\Delta}_{\ell}\right\}$, and the biorthogonality of $\Phi_{\ell}, \bar{\Phi}_{\ell}$.

Recall that if $\Phi_{\ell}$ is uniformly local and locally finite, then $\Phi_{\ell}$ is uniformly local and locally finite. Obviously, then also $\Theta_{\ell}$ is uniformly local and locally finite. It can, however, not be expected that $\tilde{\Theta}_{\ell}$ is uniformly local and locally finite, or that $\Theta_{\ell}$ (or a rescaled version) is a uniform $L_{2}(\mathcal{I})$-Riesz basis for its span. Indeed, if the latter would be true, then from the fact that $f \mapsto f^{\prime}: H_{0}^{1}(\mathcal{I}) \rightarrow L_{2}(\mathcal{I}) \cap \mathbb{1}^{\perp}$ is an isomorphism, the first relation in (5.5) would imply that $\Phi_{\ell}$ is a uniform $H^{1}(\mathcal{I})$-Riesz basis for its span, which cannot be expected for uniformly local and locally finite collection nested collections $\stackrel{+}{\Phi}_{\ell}$, with $\cup_{\ell \in \mathbb{N}} \Phi_{\ell}$ being dense in $H_{0}^{1}(\mathcal{I})$. As we will see in the next section, this fact will hamper the construction of an isotropic divergence-free wavelet Riesz basis for dimensions $n \geq 3$.

\subsection{Isotropic Divergence-Free Wavelets}

In this subsection, we discuss the construction of isotropic divergence-free wavelet bases.

In Corollary 3.12, it was shown that a Riesz basis for $\stackrel{\circ}{\mathbf{H}}^{k}\left(\mathcal{I}^{n}\right) \cap \mathbf{H}_{0}\left(\operatorname{div} 0 ; \mathcal{I}^{n}\right)$ is given by

$$
\bigcup_{\{S \subset\{1, \ldots, n\}: \# S \geq 2\}} E^{(S)} \boldsymbol{\Upsilon}^{(S)},
$$

whenever $\boldsymbol{\Upsilon}^{(S)}$ are Riesz bases for $\stackrel{\circ}{\mathbf{H}}^{k}\left(\mathcal{I}^{S}\right) \cap \mathbf{H}_{0}\left(\operatorname{div} 0 ; \mathcal{I}^{S}\right) \cap \mathbf{L}_{2,0}\left(\mathcal{I}^{S}\right)$.

W.l.o.g. considering $S=\{1, \ldots, n\}$, in Theorem 4.4 we constructed $\Upsilon^{(\{1, \ldots, n\})}$ of the form

$$
\bigcup_{\lambda \in \nabla} \mathbf{\Xi}_{\lambda}
$$

with $\boldsymbol{\Xi}_{\lambda}$ being a uniform $H^{k}\left(\mathcal{I}^{n}\right)^{n}$-Riesz basis for the $(n-1)$-dimensional space

$$
\operatorname{span}\left\{\underline{\boldsymbol{\psi}}_{\lambda, i}: 1 \leq i \leq n\right\} \cap \mathbf{H}_{0}\left(\operatorname{div} 0 ; \mathcal{I}^{n}\right)
$$

To find a single scale substitute for this basis $\Upsilon^{(\{1, \ldots, n\})}$, let us think of $\nabla$ being partitioned into a collection of finite subsets $\left\{\diamond: \diamond \in \square\right.$, and let $\boldsymbol{\Gamma}_{\diamond}$ be a uniform $H^{k}\left(\mathcal{I}^{n}\right)^{n}$-Riesz basis for 


$$
\operatorname{span}\left\{\underline{\boldsymbol{\psi}}_{\lambda, i}: \lambda \in \diamond, 1 \leq i \leq n\right\} \cap \mathbf{H}_{0}\left(\operatorname{div} 0 ; \mathcal{I}^{n}\right)
$$

Clearly, the latter space includes

$$
\bigoplus_{\lambda \in \diamond} \operatorname{span}\left\{\underline{\boldsymbol{\psi}}_{\lambda, i}: 1 \leq i \leq n\right\} \cap \mathbf{H}_{0}\left(\operatorname{div} 0 ; \mathcal{I}^{n}\right)
$$

being a space of dimension $(n-1) \times \# \diamond$. So if $\# \Gamma_{\diamond}=(n-1) \times \# \diamond$, then both spaces are equal. Since the dimension of the first space in (5.7) is equal to $n \times \# \diamond$, the condition to be verified is that its dimension is reduced by a factor $\frac{n-1}{n}$ by the intersection with $\mathbf{H}_{0}\left(\operatorname{div} 0 ; \mathcal{I}^{n}\right)$. Although we verify this in the application below, actually, using the dual wavelets, this can be proved to be always true. Anyway, in this case, $\boldsymbol{\Gamma}_{\diamond}$ provides a uniform $H^{k}\left(\mathcal{I}^{n}\right)^{n}$-Riesz basis for the space in (5.8) as an alternative for $\bigcup_{\lambda \in \diamond} \Xi_{\lambda}$. Consequently, $\bigcup_{\diamond \in \square} \boldsymbol{\Gamma}_{\diamond}$ is a Riesz basis for $\stackrel{\circ}{\mathbf{H}}^{k}\left(\mathcal{I}^{S}\right) \cap \mathbf{H}_{0}\left(\operatorname{div} 0 ; \mathcal{I}^{S}\right) \cap \mathbf{L}_{2,0}\left(\mathcal{I}^{S}\right)$ that can be used as the collection $\Upsilon^{(S)}$ in (5.6).

Again w.l.o.g. still focussing on the case $S=\{1, \ldots, n\}$, we are going to apply the above for sets $\diamond$ of the form $\left\{\lambda \in \nabla: \max _{j}\left|\lambda_{j}\right|=\ell\right\}$ for $\ell \in \mathbb{N}$. Let

$$
\begin{aligned}
& \boldsymbol{V}_{\ell}:=\operatorname{span}\left\{\underline{\boldsymbol{\psi}}_{\lambda, i}: 1 \leq i \leq n, \lambda \in \nabla, \max _{j}\left|\lambda_{j}\right|=\ell\right\}, \\
& \tilde{\boldsymbol{V}}_{\ell}:=\operatorname{span}\left\{\underline{\tilde{\boldsymbol{\psi}}}_{\lambda, i}: 1 \leq i \leq n, \lambda \in \nabla, \max _{j}\left|\lambda_{j}\right|=\ell\right\} .
\end{aligned}
$$

So $\boldsymbol{V}_{\ell}$ is the first space in (5.7) corresponding to this choice of $\diamond$, and our task is to find a uniform $H^{k}\left(\mathcal{I}^{n}\right)^{n}$-Riesz basis (i.e., $\left.\boldsymbol{\Gamma}_{\diamond}\right)$ for $\boldsymbol{V}_{\ell} \cap \mathbf{H}_{0}\left(\operatorname{div} 0 ; \mathcal{I}^{n}\right)$.

Since a Riesz basis for $\mathbf{L}_{2,0}\left(\mathcal{I}^{n}\right) \cap \stackrel{\circ}{\mathbf{H}}^{k}\left(\mathcal{I}^{n}\right)$ is given by $\left\{\left(\sum_{j=1}^{n} 4^{\mid \lambda} \mid k\right)^{-\frac{1}{2}} \underline{\boldsymbol{\psi}}_{\lambda, i}: 1 \leq\right.$ $i \leq n, \lambda \in \nabla\}$, and, thanks to Corollary 4.2, $\left\{\underline{\psi}_{\lambda, i}: 1 \leq i \leq n, \lambda \in \nabla\right\}$ is a Riesz basis for $\mathbf{L}_{2,0}\left(\mathcal{I}^{n}\right)$, from $\left(\sum_{j=1}^{n} 4^{\left|\lambda_{j}\right| k}\right)^{-\frac{1}{2}} \approx 2^{-\ell k}$ when $\max _{j}\left|\lambda_{j}\right|=\ell$, we infer that

$$
\|\cdot\|_{H^{k}\left(\mathcal{I}^{n}\right)^{n}} \approx 2^{k \ell}\|\cdot\|_{L_{2}\left(\mathcal{I}^{n}\right)^{n}} \quad \text { on } \boldsymbol{V}_{\ell}
$$

So it suffices to find a uniform $L_{2}\left(\mathcal{I}^{n}\right)^{n}$-Riesz basis for $\boldsymbol{V}_{\ell} \cap \mathbf{H}_{0}\left(\operatorname{div} 0 ; \mathcal{I}^{n}\right)$, and then to scale it with $2^{-k \ell}$.

The spaces $\boldsymbol{V}_{\ell}$ and $\tilde{\boldsymbol{V}}_{\ell}$ were defined as spans of biorthogonal anisotropic wavelets. We now construct biorthogonal isotropic wavelet bases for these spaces. The starting point are the biorthogonal univariate two-level bases $\Theta_{\ell-1}^{(i)} \cup \Psi_{\ell}^{(i)}, \tilde{\Theta}_{\ell-1}^{(i)} \cup$ $\tilde{\Psi}_{\ell}^{(i)}$ for $\operatorname{span}\left\{\psi_{\lambda}^{(i)}: \lambda \in \stackrel{\circ}{\nabla},|\lambda| \leq \ell\right\}, \operatorname{span}\left\{\tilde{\psi}_{\lambda}^{(i)}: \lambda \in \stackrel{\circ}{\nabla},|\lambda| \leq \ell\right\}$, and $\stackrel{+}{\Phi}_{\ell-1}^{(i)} \cup \dot{\Psi}_{\ell}^{(i)}$, $\widetilde{\Phi}_{\ell-1}^{(i)} \cup \widetilde{\Psi}_{\ell}^{(i)}$ for $\operatorname{span}\left\{\hat{\psi}_{\lambda}^{(i)}: \lambda \in \stackrel{\circ}{\nabla},|\lambda| \leq \ell\right\}, \operatorname{span}\left\{\widetilde{\psi}_{\lambda}^{(i)}: \lambda \in \stackrel{\circ}{\nabla},|\lambda| \leq \ell\right\}$. 
We set

$$
\begin{aligned}
& \eta_{\lambda, \delta}^{(j)}:=\left\{\begin{array}{ll}
\theta_{\lambda}^{(j)} & \text { if } \delta=0, \\
\psi_{\lambda}^{(j)} & \text { if } \delta=1,
\end{array} \tilde{\eta}_{\lambda, \delta}^{(j)}:= \begin{cases}\tilde{\theta}_{\lambda}^{(j)} & \text { if } \delta=0, \\
\tilde{\psi}_{\lambda}^{(j)} & \text { if } \delta=1,\end{cases} \right. \\
& \dot{\eta}_{\lambda, \delta}^{(j)}:=\left\{\begin{array}{ll}
\dot{\phi}_{\lambda}^{(j)} & \text { if } \delta=0, \\
\dot{+}^{(j)} & \text { if } \delta=1,
\end{array} \overline{\tilde{\eta}}_{\lambda, \delta}^{(j)}:= \begin{cases}\overline{\tilde{\phi}}_{\lambda}^{(j)} & \text { if } \delta=0, \\
\tilde{\psi}_{\lambda}^{(j)} & \text { if } \delta=1,\end{cases} \right. \\
& \triangleleft_{\ell, \delta}^{(j)}:=\left\{\begin{array}{cc}
\stackrel{\circ}{\Delta}_{\ell-1}^{(j)} & \text { if } \delta=0, \\
\left\{\lambda \in \nabla^{(j)}:|\lambda|=\ell\right\} & \text { if } \delta=1,
\end{array}\right.
\end{aligned}
$$

where $\stackrel{\circ}{\Delta}_{-1}^{(j)}:=\emptyset$. Then from Theorem 4.1 and (5.5), we have the key relations

$$
\stackrel{+}{\eta}_{\lambda, \delta}^{(j)^{\prime}}=2^{\ell} \eta_{\lambda, \delta}^{(j)}, \quad \tilde{\eta}_{\lambda, \delta}^{(j)^{\prime}}=-2^{\ell} \overline{\tilde{\eta}}_{\lambda, \delta}^{(j)} \quad\left(\lambda \in \triangleleft_{\ell, 0}^{(j)} \cup \triangleleft_{\ell, 1}^{(j)}\right) .
$$

With

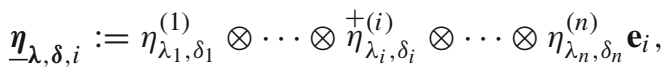

$$
\begin{aligned}
& \underline{\tilde{\eta}}_{\lambda, \delta, i}:=\tilde{\eta}_{\lambda_{1}, \delta_{1}}^{(1)} \otimes \cdots \otimes \overline{\tilde{\eta}}_{\lambda_{i}, \delta_{i}}^{(i)} \otimes \cdots \otimes \tilde{\eta}_{\lambda_{n}, \delta_{n}}^{(n)} \mathbf{e}_{i},
\end{aligned}
$$

biorthogonal isotropic wavelet bases for $\boldsymbol{V}_{\ell}$ and $\tilde{\boldsymbol{V}}_{\ell}$ are now given by

$$
\begin{aligned}
& \left\{\underline{\eta}_{\lambda, \delta, i}: \overrightarrow{0} \neq \delta \in\{0,1\}^{n}, \lambda \in \prod_{j=1}^{n} \triangleleft_{\ell, \delta_{j}}^{(j)}, 1 \leq i \leq n\right\}, \\
& \left\{\underline{\tilde{\eta}}_{\lambda, \delta, i}: \overrightarrow{0} \neq \delta \in\{0,1\}^{n}, \lambda \in \prod_{j=1}^{n} \triangleleft_{\ell, \delta_{j}}^{(j)}, 1 \leq i \leq n\right\} .
\end{aligned}
$$

Next, analogously to Theorem 4.4, we are going to transform these bases to identify divergence-free primal wavelets, and dual wavelets that are gradients. Let $\mathbf{Q} \in \mathbb{R}^{n \times n}$ be orthogonal with its $n$th row given by $[1 \ldots 1] / \sqrt{n}$. We define

$$
\left[\begin{array}{c}
\eta_{\lambda, \delta, 1} \\
\vdots \\
\eta_{\lambda, \delta, n}
\end{array}\right]:=\mathbf{Q}\left[\begin{array}{c}
\underline{\eta}_{\lambda, \delta, 1} \\
\vdots \\
\underline{\eta}_{\lambda, \delta, n}
\end{array}\right], \quad\left[\begin{array}{c}
\tilde{\eta}_{\lambda, \delta, 1} \\
\vdots \\
\tilde{\eta}_{\lambda, \delta, n}
\end{array}\right]:=\mathbf{Q}\left[\begin{array}{c}
\underline{\tilde{\eta}}_{\lambda, \delta, 1} \\
\vdots \\
\underline{\tilde{\eta}}_{\lambda, \delta, n}
\end{array}\right] .
$$


Proposition 5.4 Biorthogonal bases for $\boldsymbol{V}_{\ell}$ and $\tilde{\boldsymbol{V}}_{\ell}$ are given by

$$
\begin{aligned}
& \boldsymbol{\Psi}_{\text {iso }, \ell}:=\left\{\eta_{\lambda, \delta, i}: \overrightarrow{0} \neq \delta \in\{0,1\}^{n}, \lambda \in \prod_{j=1}^{n} \triangleleft_{\ell, \delta_{j}}^{(j)}, 1 \leq i \leq n\right\}, \\
& \tilde{\boldsymbol{\Psi}}_{\text {iso }, \ell}:=\left\{\tilde{\eta}_{\lambda, \delta, i}: \overrightarrow{0} \neq \delta \in\{0,1\}^{n}, \lambda \in \prod_{j=1}^{n} \triangleleft_{\ell, \delta_{j}}^{(j)}, 1 \leq i \leq n\right\} .
\end{aligned}
$$

Furthermore, for $1 \leq i \leq n-1, \eta_{\lambda, \delta, i} \in \mathbf{H}_{0}\left(\operatorname{div} 0 ; \mathcal{I}^{n}\right)$, and $\tilde{\eta}_{\lambda, \delta, n} \in \operatorname{grad} H^{1}\left(\mathcal{I}^{n}\right)$.

Proof For $1 \leq i \leq n-1$, it holds that

$$
\operatorname{div} \boldsymbol{\eta}_{\lambda, \delta, i}=\sum_{j=1}^{n} \mathbf{Q}_{i j} \operatorname{div} \underline{\boldsymbol{\eta}}_{\lambda, \boldsymbol{\delta}, i} \stackrel{(5.10)}{=}\left(\sum_{j=1}^{n} \mathbf{Q}_{i j} 2^{\ell}\right) \eta_{\lambda_{1}, \delta_{1}}^{(1)} \otimes \cdots \otimes \eta_{\lambda_{n}, \delta_{n}}^{(n)}=0,
$$

and

$$
\sqrt{n} \tilde{\eta}_{\lambda, \delta, n}=\sum_{i=1}^{n} \tilde{\eta}_{\lambda, \delta, i} \stackrel{(5.10)}{=}-2^{-\ell} \operatorname{grad} \tilde{\eta}_{\lambda_{1}, \delta_{1}}^{(1)} \otimes \cdots \otimes \tilde{\eta}_{\lambda_{n}, \delta_{n}}^{(n)}
$$

Consequently,

$$
\Psi_{\text {iso }, \ell}^{(\mathrm{df})}:=\left\{\eta_{\lambda, \delta, i}: \overrightarrow{0} \neq \delta \in\{0,1\}^{n}, \lambda \in \prod_{j=1}^{n} \triangleleft_{\ell, \delta_{j}}^{(j)}, 1 \leq i \leq n-1\right\}
$$

is a basis for $\boldsymbol{V}_{\ell} \cap \mathbf{H}_{0}\left(\operatorname{div} 0 ; \mathcal{I}^{n}\right)$, and apparently $\frac{\operatorname{dim} \boldsymbol{V}_{\ell} \cap \mathbf{H}_{0}\left(\operatorname{div} 0 ; \mathcal{I}^{n}\right)}{\operatorname{dim} \boldsymbol{V}_{\ell}}=\frac{n-1}{n}$ as required. We conclude that, possibly after an $L_{2}\left(\mathcal{I}^{n}\right)^{n}$-stabilization of $\boldsymbol{\Psi}_{\text {iso, } \ell}^{(\mathrm{df})}$, by Gram-Schmidt or otherwise, so that it becomes a uniform (in $\ell$ ) $L_{2}\left(\mathcal{I}^{n}\right)^{n}$-Riesz basis of its span, the collection

$$
\sum_{\ell \in \mathbb{N}} 2^{-k \ell} \boldsymbol{\Psi}_{\text {iso }, \ell}^{(\mathrm{df})}
$$

can serve as the Riesz basis $\boldsymbol{\Upsilon}^{(S)}$ for $\stackrel{\circ}{\mathbf{H}}^{k}\left(\mathcal{I}^{S}\right) \cap \mathbf{H}_{0}\left(\operatorname{div} 0 ; \mathcal{I}^{S}\right) \cap \mathbf{L}_{2,0}\left(\mathcal{I}^{S}\right)$ as needed in (5.6) (here for $S=\{1, \ldots, n\}$ ).

Unfortunately, due to the presence of factors $\theta_{\lambda}^{(j)}$ in the definition of $\underline{\boldsymbol{\eta}}_{\lambda, \boldsymbol{\delta}, i}$, and the fact that the $\Theta_{\ell}^{(j)}$ are not uniform $L_{2}(\mathcal{I})$-Riesz bases for their spans, for $n \geq 3$, the aforementioned $L_{2}\left(\mathcal{I}^{n}\right)^{n}$-stabilization of $\Psi_{\text {iso, } \ell}^{(\mathrm{df})}$ is required, and it spoils locality of the basis functions.

As we will show below, for $n=2$, however, $\Psi_{\text {iso, }, \ell}^{(\mathrm{df})}$ is already an $L_{2}\left(\mathcal{I}^{n}\right)^{n}$-Riesz basis of its span, and a further stabilization is not required. Related to this is the fact that 
for $n=2$, we can use the freedom in the construction of a dual basis (cf. Remark 4.6) such that it becomes a local one, despite the fact that $\tilde{\Theta}_{\ell}$ is not local.

Theorem 5.5 For $i \in\{1,2\}$, consider the situation as in Theorem 4.1. Let $\Psi^{(i)}, \tilde{\Psi}^{(i)}$, and so $\Psi^{(i)}, \widetilde{\Psi}^{(i)}$, be local. In the situation as in Proposition 5.1, let $\Phi_{\ell}^{(i)}, \tilde{\Phi}_{\ell}^{(i)}$, and so $\Phi_{\ell}^{(i)}, \tilde{\Phi}_{\ell}^{(i)}$, and $\Theta_{\ell}^{(i)}$ defined in (5.4), be uniformly local and locally finite. Then

$$
\begin{aligned}
& \bigcup_{\ell \in \mathbb{N}} 2^{-\ell k}\left\{\left\{\stackrel{\psi}{\psi}_{\lambda_{1}}^{(1)} \otimes \psi_{\lambda_{2}}^{(2)} \mathbf{e}_{1}-\psi_{\lambda_{1}}^{(1)} \otimes \stackrel{+}{\psi}_{\lambda_{2}}^{(2)} \mathbf{e}_{2}:\left(\lambda_{1}, \lambda_{2}\right) \in \stackrel{\circ}{\nabla}^{(1)} \times \stackrel{\circ}{\nabla^{(2)}},\left|\lambda_{1}\right|=\left|\lambda_{2}\right|=\ell\right\}\right. \\
& \cup\left\{\stackrel{+}{\psi}_{\lambda_{1}}^{(1)} \otimes \theta_{\lambda_{2}}^{(2)} \mathbf{e}_{1}-\psi_{\lambda_{1}}^{(1)} \otimes \stackrel{+}{\phi}_{\lambda_{2}}^{(2)} \mathbf{e}_{2}:\left(\lambda_{1}, \lambda_{2}\right) \in \stackrel{\circ}{\nabla}^{(1)} \times \stackrel{\circ}{\Delta}_{\ell-1}^{(2)},\left|\lambda_{1}\right|=\ell\right\} \\
& \left.\cup\left\{\stackrel{+}{\phi}_{\lambda_{1}}^{(1)} \otimes \psi_{\lambda_{2}}^{(2)} \mathbf{e}_{1}-\theta_{\lambda_{1}}^{(1)} \otimes \stackrel{+}{\psi}_{\lambda_{2}}^{(2)} \mathbf{e}_{2}:\left(\lambda_{1}, \lambda_{2}\right) \in \stackrel{\circ}{\Delta}_{\ell-1}^{(1)} \times \stackrel{\circ}{\nabla^{(2)}},\left|\lambda_{2}\right|=\ell\right\}\right\}
\end{aligned}
$$

is a Riesz basis for $\stackrel{\circ}{\mathbf{H}}^{k}\left(\mathcal{I}^{2}\right) \cap \mathbf{H}_{0}\left(\operatorname{div} 0 ; \mathcal{I}^{2}\right)$, with dual basis

$$
\begin{aligned}
\bigcup_{\ell \in \mathbb{N}} 2^{\ell k}\left\{\left\{\frac{\tilde{\psi}_{\lambda_{1}}^{(1)} \otimes \tilde{\psi}_{\lambda_{2}}^{(2)} \mathbf{e}_{1}-\tilde{\psi}_{\lambda_{1}}^{(1)} \otimes \tilde{\psi}_{\lambda_{2}}^{(2)} \mathbf{e}_{2}}{\sqrt{2}}:\left(\lambda_{1}, \lambda_{2}\right) \in \stackrel{\circ}{\nabla}^{(1)} \times \stackrel{\circ}{\nabla}^{(2)},\left|\lambda_{1}\right|=\left|\lambda_{2}\right|=\ell\right\}\right. \\
\cup\left\{\tilde{\psi}_{\lambda_{1}}^{(1)} \otimes \widetilde{\phi}_{\lambda_{2}}^{(2)} \mathbf{e}_{2}:\left(\lambda_{1}, \lambda_{2}\right) \in \stackrel{\circ}{\nabla}^{(1)} \times \stackrel{\circ}{\Delta}_{\ell-1}^{(2)},\left|\lambda_{1}\right|=\ell\right\} \\
\left.\cup\left\{\widetilde{\phi}_{\lambda_{1}}^{(1)} \otimes \tilde{\psi}_{\lambda_{2}}^{(2)} \mathbf{e}_{1}:\left(\lambda_{1}, \lambda_{2}\right) \in \stackrel{\circ}{\Delta}_{\ell-1}^{(1)} \times \stackrel{\circ}{\nabla}^{(2)},\left|\lambda_{2}\right|=\ell\right\}\right\} .
\end{aligned}
$$

Proof Let us denote the primal and dual collections as $\bigcup_{\ell \in \mathbb{N}} 2^{-\ell k}\left\{\Psi_{\ell}^{(11)} \cup \Psi_{\ell}^{(10)} \cup\right.$ $\left.\boldsymbol{\Psi}_{\ell}^{(01)}\right\}$ and $\bigcup_{\ell \in \mathbb{N}} 2^{\ell k}\left\{\tilde{\boldsymbol{\Psi}}_{\ell}^{(11)} \cup \tilde{\boldsymbol{\Psi}}_{\ell}^{(10)} \cup \tilde{\boldsymbol{\Psi}}_{\ell}^{(01)}\right\}$.

The biorthogonality follows from the biorthogonality of the pairs $\left(\Psi^{(i)}, \tilde{\Psi}^{(i)}\right)$, $\left(\overleftarrow{\Psi}^{(i)}, \tilde{\Psi}^{(i)}\right), \quad\left(\Phi_{\ell}^{(i)}, \tilde{\Phi}_{\ell}^{(i)}\right), \quad\left(\stackrel{\Phi}{\Phi}_{\ell}^{(i)}, \tilde{\Phi}_{\ell}^{(i)}\right), \quad$ and from $\operatorname{span} \overleftarrow{\Phi}_{\ell}^{(i)}=\operatorname{span}\left\{\dot{\psi}_{\lambda}^{(i)}: \lambda\right.$ $\left.\in \stackrel{\circ}{\nabla}^{(i)},|\lambda| \leq \ell\right\}, \operatorname{span} \widetilde{\Phi}_{\ell}^{(i)}=\operatorname{span}\left\{\widetilde{\psi}_{\lambda}^{(i)}: \lambda \in \stackrel{\circ}{\nabla}^{(i)},|\lambda| \leq \ell\right\}$, and $\operatorname{span} \Theta_{\ell}^{(i)}=$ $\operatorname{span}\left\{\psi_{\lambda}^{(i)}: \lambda \in \stackrel{\circ}{\nabla}^{(i)},|\lambda| \leq \ell\right\}$.

Apart from a harmless factor $\sqrt{2}$, the primal collection is equal to that from (5.11). Since for $n=2,(5.6)$ reads as $\Upsilon^{(\{1,2\})}$, in order to conclude that the primal collection is a Riesz basis for $\mathbf{H}^{k}\left(\mathcal{I}^{2}\right) \cap \mathbf{H}_{0}\left(\operatorname{div} 0 ; \mathcal{I}^{2}\right)$, in view of the preceding analysis in this subsection, what remains to show is that $\bigcup_{0 \neq \delta \in\{0,1\}^{2}} \Psi_{\ell}^{(\boldsymbol{\delta})}$ is a uniform $L_{2}\left(\mathcal{I}^{2}\right)$-Riesz basis for its span.

From the collections being uniformly local and locally finite, and the functions having uniformly bounded $L_{2}\left(\mathcal{I}^{2}\right)^{2}$-norms, it follows that $\mathbf{u}_{\ell}:=\sum_{0 \neq \delta \in\{0,1\}^{2}} \mathbf{c}_{\ell}^{(\boldsymbol{\delta})^{\top}} \Psi_{\ell}^{(\boldsymbol{\delta})}$ satisfies $\left\|\mathbf{u}_{\ell}\right\|_{L_{2}\left(\mathcal{I}^{2}\right)}^{2} \lesssim \sum_{0 \neq \delta \in\{0,1\}^{2}}\left\|\mathbf{c}^{(\boldsymbol{\delta})}\right\|_{\ell_{2}}^{2}$. Below we will show that the dual collection is a dual basis in the sense of (1.7). Then, $\left\|\mathbf{c}^{(\boldsymbol{\delta})}\right\|_{\ell_{2}}=\left\|\left\langle\mathbf{u}_{\ell}, \tilde{\boldsymbol{\Psi}}_{\ell}^{(\boldsymbol{\delta})}\right\rangle_{L_{2}\left(\mathcal{I}^{2}\right)^{2}}\right\|_{\ell_{2}} \lesssim$ $\left\|2^{-k \ell} \mathbf{u}_{\ell}\right\|_{H^{k}\left(\mathcal{I}^{2}\right)^{2}} \lesssim\left\|\mathbf{u}_{\ell}\right\|_{L_{2}\left(\mathcal{I}^{2}\right)^{2}}$ by (5.9), with which the proof is completed.

Since, up to a harmless difference in scaling, $\bigcup_{\ell \in \mathbb{N}} 2^{\ell k} \tilde{\boldsymbol{\Psi}}_{\ell}^{(11)}$ is a subset of the collection in (4.11), we infer that for $\mathbf{u} \in H^{k}\left(\mathcal{I}^{2}\right)^{2}, \sum_{\ell \in \mathbb{N}}\left\|\left\langle\mathbf{u}, 2^{\ell k} \tilde{\boldsymbol{\Psi}}_{\ell}^{(11)}\right\rangle_{L_{2}\left(\mathcal{I}^{2}\right)^{2}}\right\|_{\ell_{2}}^{2} \lesssim$ $\|\mathbf{u}\|_{H^{k}\left(\mathcal{I}^{2}\right)^{2}}^{2}$ 
To show that the dual collection is a dual basis, it remains to prove that for $\mathbf{u} \in$ $H^{k}\left(\mathcal{I}^{2}\right)^{2}$,

$$
\sum_{\ell \in \mathbb{N}}\left\|\left\langle\mathbf{u}, 2^{\ell k} \tilde{\boldsymbol{\Psi}}_{\ell}^{(10)}\right\rangle_{L_{2}\left(\mathcal{I}^{2}\right)^{2}}\right\|_{\ell_{2}}^{2} \lesssim\left\|\mathbf{u} \cdot \mathbf{e}_{2}\right\|_{H^{k}\left(\mathcal{I}^{2}\right)}^{2},
$$

because the proof for $\tilde{\boldsymbol{\Psi}}_{\ell}^{(10)}$ reading as $\tilde{\boldsymbol{\Psi}}_{\ell}^{(01)}$ is similar.

From $\Psi^{(1)}$ and $\left\{2^{-|\lambda| k} \psi_{\lambda}^{(1)}: \lambda \in \nabla^{(1)}\right\}$ being Riesz bases for $L_{2}(\mathcal{I})$ and $H_{\left(t_{0}^{(1)}, t_{1}^{(1)}\right)}^{k}(\mathcal{I})$, respectively, and $\stackrel{+}{\Psi}^{(2)}$ and $\left\{2^{-|\lambda| k} \stackrel{+}{\psi}_{\lambda}^{(2)}: \lambda \in \stackrel{\circ}{\nabla}^{(2)}\right\}$ being Riesz bases for $L_{2}(\mathcal{I})$ and $H_{\left(n_{0}^{(2)}, n_{1}^{(2)}\right)}^{k}(\mathcal{I})$, respectively, we have that

$$
\begin{gathered}
\left\{\left(\sum_{j=1}^{2} 4^{\left|\lambda_{j}\right| k}\right)^{-\frac{1}{2}} \psi_{\lambda_{1}}^{(1)} \otimes \stackrel{+}{\psi}{ }_{\lambda_{2}}^{(2)}:\left(\lambda_{1}, \lambda_{2}\right) \in \nabla^{(1)} \times \stackrel{\circ}{\nabla}^{(1)}\right\} \text { is a Riesz basis for } \\
\left\{\mathbf{u} \cdot \mathbf{e}_{2}: \mathbf{u} \in \stackrel{\circ}{H}^{k}\left(\mathcal{I}^{2}\right)\right\} \simeq H_{\left(t_{0}^{(1)}, t_{1}^{(1)}\right)}^{k}(\mathcal{I}) \otimes L_{2}(\mathcal{I}) \cap L_{2}(\mathcal{I}) \otimes H_{\left(n_{0}^{(2)}, n_{1}^{(2)}\right)}^{k}(\mathcal{I})
\end{gathered}
$$

Since $\left\{\psi_{\lambda_{1}}^{(1)} \otimes \stackrel{+}{\psi}_{\lambda_{2}}^{(2)}:\left(\lambda_{1}, \lambda_{2}\right) \in \nabla^{(1)} \times \stackrel{\circ}{\nabla}^{(2)}\right\}$ is a Riesz basis for $L_{2}\left(\mathcal{I}^{2}\right)$, we have for $\ell \in \mathbb{N}$,

$$
\begin{aligned}
& \|\cdot\|_{H^{k}\left(\mathcal{I}^{2}\right)} \approx 2^{k \ell}\|\cdot\|_{L_{2}\left(\mathcal{I}^{2}\right)} \quad \text { on } \\
& \operatorname{span}\left\{\psi_{\lambda_{1}}^{(1)} \otimes \stackrel{+}{\psi}{ }_{\lambda_{2}}^{(2)}:\left(\lambda_{1}, \lambda_{2}\right) \in \nabla^{(1)} \times \stackrel{\circ}{\nabla^{(2)}}, \max \left(\left|\lambda_{1}\right|,\left|\lambda_{2}\right|\right)=\ell\right\} .
\end{aligned}
$$

An alternative, uniform $L_{2}\left(\mathcal{I}^{2}\right)$-basis for the space from (5.14) is given by

$$
\Psi_{\ell}^{(1)} \otimes \Phi_{\ell-1}^{(2)} \cup \Phi_{\ell-1}^{(1)} \otimes \Psi_{\ell}^{(2)} \cup \Psi_{\ell}^{(1)} \otimes \overleftarrow{\Psi}_{\ell}^{(2)}
$$

The latter result, (5.14), and (5.13) show that

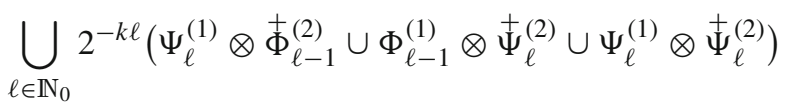

is a Riesz basis for $\left\{\mathbf{u} \cdot \mathbf{e}_{2}: \mathbf{u} \in \stackrel{\circ}{\mathbf{H}}^{k}\left(\mathcal{I}^{2}\right)\right\}$.

Its unique dual basis in $L_{2}\left(\mathcal{I}^{2}\right)$ reads as

$$
\bigcup_{\ell \in \mathbb{N}} 2^{k \ell}\left(\tilde{\Psi}_{\ell}^{(1)} \otimes \widetilde{\Phi}_{\ell-1}^{(2)} \cup \tilde{\Phi}_{\ell-1}^{(1)} \otimes \tilde{\Psi}_{\ell}^{(2)} \cup \tilde{\Psi}_{\ell}^{(1)} \otimes \tilde{\Psi}_{\ell}^{(2)}\right) .
$$

Since $\tilde{\Psi}_{\ell}^{(10)}=\tilde{\Psi}_{\ell}^{(1)} \otimes \widetilde{\Phi}_{\ell-1}^{(2)} \mathbf{e}_{2}$, we conclude (5.12), which completes the proof.

\section{Conclusion}

Anisotropic wavelet Riesz bases were constructed for the space of divergence-free vector fields on the $n$-dimensional hypercube that have vanishing normal components, 
or for this space intersected with higher order Sobolev spaces that may incorporate additional boundary conditions, e.g., no-slip boundary conditions. The construction relies on a principle introduced by Lemarié-Rieusset in [21] for the construction of divergence-free wavelet bases on $\mathbb{R}^{n}$, but only after making an initial biorthogonal decomposition of $\left.\left.\left(L_{2}(0,1)^{n}\right)^{n}, L_{2}(0,1)^{n}\right)^{n}\right)$ into $2^{n}-1$ pairs of subspaces. For $n=2$, an alternative isotropic wavelet basis was constructed.

Open Access This article is distributed under the terms of the Creative Commons Attribution 4.0 International License (http://creativecommons.org/licenses/by/4.0/), which permits unrestricted use, distribution, and reproduction in any medium, provided you give appropriate credit to the original author(s) and the source, provide a link to the Creative Commons license, and indicate if changes were made.

\section{References}

1. Balder, R., Zenger, Ch.: The solution of multidimensional real Helmholtz equations on sparse grids. SIAM J. Sci. Comput. 17(3), 631-646 (1996)

2. Battle, G., Federbush, P.: Divergence-free vector wavelets. Mich. Math. J. 40(1), 181-195 (1993)

3. Černá, D., Finěk, V.: Cubic spline wavelets with complementary boundary conditions. Appl. Math. Comput. 219(4), 1853-1865 (2012)

4. Cohen, A., Daubechies, I., Feauveau, J.C.: Biorthogonal bases of compactly supported wavelets. Commun. Pure Appl. Math. 45, 485-560 (1992)

5. Dahmen, W., Schneider, R.: Wavelets with complementary boundary conditions-function spaces on the cube. Results Math. 34(3-4), 255-293 (1998)

6. Dauge, M., Stevenson, R.P.: Sparse tensor product wavelet approximation of singular functions. SIAM J. Math. Anal. 42(5), 2203-2228 (2010)

7. Deriaz, E., Domingues, M.O., Perrier, V., Schneider, K., Farge, M.: Divergence-free wavelets for coherent vortex extraction in 3D homogeneous isotropic turbulence. In: CEMRACS 2005-Computational Aeroacoustics and Computational Fluid Dynamics in Turbulent Flows, volume 16 of ESAIM Proceedings, pp. 146-163. EDP Sci., Les Ulis (2007)

8. Deriaz, E., Perrier, V.: Divergence-free and curl-free wavelets in two dimensions and three dimensions: application to turbulent flows. J. Turbul. 7. Paper 3 (electronic) (2006). doi:10.1080/ 14685240500260547

9. Deriaz, E., Perrier, V.: Direct numerical simulation of turbulence using divergence-free wavelets. Multiscale Model. Simul. 7(3), 1101-1129 (2008)

10. Dijkema, T.J.: Adaptive tensor product wavelet methods for solving PDEs. Ph.D. thesis, Utrecht University (2009)

11. Girault, V., Raviart, P.A.: Finite Element Methods for Navier-Stokes Equations. Theory and Algorithms. Springer, Berlin (1986)

12. Griebel, M., Knapek, S.: Optimized tensor-product approximation spaces. Constr. Approx. 16(4), 525540 (2000)

13. Griebel, M., Oswald, P.: Tensor product type subspace splittings and multilevel iterative methods for anisotropic problems. Adv. Comput. Math. 4(1-2), 171-206 (1995)

14. Jouini, A., Lemarié-Rieusset, P.G.: Analyse multi-résolution bi-orthogonale sur l'intervalle et applications. Ann. Inst. H. Poincaré Anal. Non Linéaire 10(4), 453-476 (1993)

15. Kadri, HarounaS, Perrier, V.: Effective construction of divergence-free wavelets on the square. J. Comput. Appl. Math. 240, 74-86 (2013)

16. Kadri, HarounaS, Perrier, V.: Divergence-free wavelet projection method for incompressible viscous flow on the square. Multiscale Model. Simul. 13(1), 399-422 (2015)

17. Kestler, S., Stevenson, R.P.: Fast evaluation of system matrices w.r.t. multi-tree collections of tensor product refinable basis functions. J. Comput. Appl. Math. 260, 103-116 (2014)

18. Lakey, J.D., Nguyen, P.: Divergence-free multiwavelets on the half plane. Axioms 2, 100-121 (2013) 
19. Lakey, J.D., Pereyra, M.C.: Divergence-free multiwavelets on rectangular domains. In: Wavelet Analysis and Multiresolution Methods (Urbana-Champaign, IL, 1999), volume 212 of Lecture Notes in Pure and Applied Mathematics, pp. 203-240. Dekker, New York (2000)

20. Lakey, J.D., Pereyra, M.C.: On the nonexistence of certain divergence-free multiwavelets. In: Wavelets and Signal Processing, Appl. Numer. Harmon. Anal., pp. 41-54. Birkhäuser Boston, Boston (2003)

21. Lemarié-Rieusset, P.G.: Analyses multi-résolutions non orthogonales, commutation entre projecteurs et dérivation et ondelettes vecteurs à divergence nulle. Rev. Math. Iberoam. 8(2), 221-237 (1992)

22. Lemarié-Rieusset, P.G.: Un théorème d'inexistence pour les ondelettes vecteurs à divergence nulle. C. R. Acad. Sci. Paris Sér. I Math. 319(8), 811-813 (1994)

23. Primbs, M.: New stable biorthogonal spline-wavelets on the interval. Results Math. 57(1-2), 121-162 (2010)

24. Stevenson, R.P.: Divergence-free wavelets on the hypercube. Appl. Comput. Harmon. Anal. 30, 1-19 (2011)

25. Stevenson, R.P.: Divergence-free wavelets on the hypercube: free-slip boundary conditions, and applications for solving the instationary Stokes equations. Math. Comput. 80, 1499-1523 (2011)

26. Urban, K.: Using divergence free wavelets for the numerical solution of the Stokes problem. In: Axelsson, O., Polman, B. (eds.) Algebraic Multilevel Iteration Methods with Applications, pp. 261278. University of Nijmegen, Nijmegen (1996)

27. Urban, K.: Wavelet bases in $H$ (div) and $H$ (curl). Math. Comput. 70(234), 739-766 (2001)

28. Urban, K.: Wavelets in Numerical Simulation, volume 22 of Lecture Notes in Computational Science and Engineering. Springer, Berlin (2002). Problem adapted construction and applications

29. Zhou, X., He, Y.: Using divergence free wavelets for the numerical solution of the 2-D stationary Navier-Stokes equations. Appl. Math. Comput. 163(2), 593-607 (2005) 